\title{
The normal gravity formula and the polar flattening according to geodetic reference system 1967
}

\author{
M. Ciputo $(*)\left(^{* *}\right)-$ I. PIERI $\left(^{* *}\right)$ \\ Ricevuto il 6 Aprile 1968
}

Riassunto. - Scopo del presente lavoro è di fornire i valori dello schiacciamento $f$ dellellissoide di rotazione terrestre e di coeflicienti della gravità normale g deducendoli dai valori adottati dall'Assemblea Generale di Geodesia e Geofisica del 1967, tenendo conto della massa dell atmosfera e facendo uso delle formule chiuse della teoria di Pizzetti-Somirliana che compaiono in: MI. Caputo, "The Gravity Field of the Earth from classical and Modern Methods", Acarlemic Press, 1967.

Si trova

$$
\begin{aligned}
& f=(298.247047)^{-1} \\
& g=978.03101 \frac{1+0.00193167 \sin ^{2} \phi}{\left[1-0.0066\left(69461 \mathrm{sin}^{2} \mathrm{~m}^{1 / 1 / 2}\right.\right.} .
\end{aligned}
$$

Segue una tavola dei valori del modulo della gravità per la latitudine variabile da $0^{\circ}$ a $90^{\circ}$ con passo di $1^{\prime}$.

SUMmar. - We computed the values of fattening $f$ of the equipotential ellipsoid of the Farth's field and of coefficients of the normal gravity, according to the Larth's parameters adopted by the International Union of Geodesy and Geophysies at its General Assembly of 1967 taling into account the mass of atmosphere and using the closed formulae of the Pizzetti-Somigliana theory that appear in: M. Caputo "The Gravity Field of the Earth from classical and modern Methods", Acarlemic Press, 1967.

We obtained

$$
\begin{aligned}
& f=(298.247047)^{-1} \\
& g=978.03101 \frac{1+0.00193167 \sin ^{2} \varphi}{\left[1-0.00669461 \sin ^{2} \varphi\right]^{1 / 2}} .
\end{aligned}
$$

We added a table of values of modulus of the gravity vector for $q$ varying from $0^{\circ}$ to $90^{\circ}$ with a pass of $1^{\prime}$.

(*) Istituto di Fisica dell'Università di Bologna.

$\left(^{* *}\right)$ Istituto di Geodesia dell'Università di Bologna. 


\section{ERRATA CORRIGE:}

Dal volume XXI 1.. 1 - 1968 - Articolo M. Caputo - L. Pieri:

Pg. 123 lines 3 and 15 - pg. 124 lines 1 and 5 from top substitute "recommended" with "adopted". 
The International Astronomical Union in 1964 adopted for the system of astronomical constants the ralues of the Earth's parameters surgesterl by the Commission of International Union of Geodesy. The International Lnion of Geodesy and Geophysics, at its General Assembly of 1967 , arlopted the same parameters the values of which are:

Erjuatorial rarlius

Coefficient of second order zonal harmonic of the potential

Product of mass of Earth and atmosphere times gravitational constant

Ratio mass of atmosphere to mass of Earth

$$
\begin{aligned}
& a_{1}=6.378160 \cdot 10^{8} \mathrm{~cm} \\
& C_{20}=-1.08270 \cdot 10^{-3}
\end{aligned}
$$

The purpose of this work is of setting up the polar flattening, $f$, and the coefficients in the formula for the normal gravity field.

We assume that the normal figure of the Earth is an ellipsoid of revolution. In this hypothesis the Pizzetti-Somigliana theory allows us to express the flattening $f$ and the normal gravity $\left({ }^{3}\right)$, with closed formulae ( $\left.{ }^{1}\right)$.

For the flattening we have $\left({ }^{2}\right)$ :

$$
\begin{gathered}
W=\frac{M G}{r}\left\{1+\sum_{1}^{\infty}\left(\frac{a_{1}}{r}\right)^{2 n} C_{2 n o} P_{2 n}(\cos \vartheta)\right\}, \\
C_{2 n}=\frac{(-1)^{n}}{2 n+1}\left|1+\frac{8 n K_{0}}{3(2 n+3) M}\right| f^{n}(2-f)^{n},
\end{gathered}
$$

and for our neerls:

$$
C_{20}=+\frac{1}{3}\left|1-\frac{4 \varepsilon^{3} a_{3}^{2}\left(1+\varepsilon^{2}\right) \omega^{2}}{15 M G\left\{\left(3+\varepsilon^{2}\right) \operatorname{arctg} \varepsilon-3 \varepsilon\right\}}\right|(f-2) f,
$$

with:

$$
\varepsilon^{2}=\frac{a_{1}^{2}-a_{3}^{2}}{a_{3}^{2}}-\frac{f(2-f)}{(1-f)^{2}}=\sum_{0}^{\infty}(2+n) f^{n+1}
$$

second eccentricity. 
THE NORMAL GRAVITY FORMULA AND THE POLAR FJ.ATTENIXg, ETC. 125

Where $a_{3}=a_{1}(1-f)$ is the polar radius of the normal ellipsoid; and we assume the Earth's rotation rate:

$$
\omega=0.72921151 \cdot 10^{-1} \mathrm{sec}^{-1} .
$$

The Pizzetti and Clairaut theorems are $\left(^{1}\right)$ :

$$
\begin{aligned}
& g_{a 3}=\frac{a_{3} g_{a 1}}{a_{1}}+\omega^{2} a_{3}\left[\begin{array}{l}
\frac{3}{2}+\frac{(n+1) \varepsilon^{2 n}(-1)^{n}}{2} \\
2 \frac{\varepsilon^{2}}{\sum_{0}^{\infty}} \frac{(n+1) \varepsilon^{2 n}(-1)^{n}}{(2 n+3)(2 n+5)}
\end{array}\right] \\
& 2 \omega^{2} a_{1}^{2} a_{3}+2 g_{a 1} a_{1} a_{3}+g_{a 3} a_{1}^{2}=3 G M,
\end{aligned}
$$

where:

$g_{a 1}$ is the acceleration of gravity at the equator, $g_{a 3}$ is the acceleration of gravity at the Earth's pole.

We can compute $g_{a 1}$ and $g_{a 3}$ using [3] and [4].

The closed expression of the normal gravity is ${ }^{(1)}$ :

$$
g=g_{\alpha 1} \frac{1+(\alpha-f-f \alpha) \sin ^{2} \varphi}{\left[1-f(2-f) \sin ^{2} \varphi\right]^{1 / 2}}
$$

where:

$$
\alpha=\frac{g_{a 3}-g_{a 1}}{g_{a 1}} .
$$

For the numerical computation of $f$ from [2'] we have:

$$
\begin{aligned}
& f^{3}-f^{2}\left[\frac{15 M G \sum_{n}^{\infty} \frac{\left(-1 j^{n} \varepsilon^{2 n}(n+1)\right.}{(2 n+3)(2 n+5)}}{a_{1}^{3} \omega^{2}}+3\right]+ \\
& +f\left[3+\frac{30 M G}{a_{1}^{2} \omega^{2}} \sum_{0}^{\infty} \frac{(-1)^{n} \varepsilon^{2 n}(n+1)}{(2 n+3)(2 n+5)}\right]+ \\
& +\frac{45 M G C_{20}^{\infty}}{x_{1}^{5} \omega^{2}} \frac{(-1)^{n} \varepsilon^{2 n}(n+1)}{(2 n+3)(2 n+5)}-1=0
\end{aligned}
$$

which gives with the adopted values [1]:

$$
f^{-1}=298.247047 \text {. }
$$


For the numerical computation of $g_{a 1}$ and $g_{a 3}$ we have from [2] and [3]:

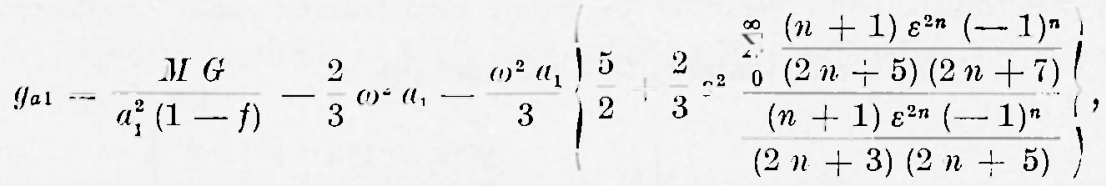

$$
\begin{aligned}
& g_{a 3}=\frac{M G}{a_{1}^{2}}-\frac{2}{3} \omega^{2} a_{1}(1-f)+\frac{2}{3} \omega^{2} a_{1}(1-f) \\
& \left\{\frac{5}{2}-\frac{3}{2} \varepsilon^{2} \frac{\sum_{0}^{\infty} \frac{(n+1) \varepsilon^{2 n}(-1)^{n}}{(2 n+5)(2 n+7)}}{\sum_{0}^{\infty} \frac{(n+1) \varepsilon^{2 n}(-1)^{n}}{(2 n+3)(2 n+5)}}\right\}
\end{aligned}
$$

Using the above-mentioned numerical values [1] we obtain:

$$
\begin{aligned}
& g_{a 1}=978.03101 \\
& g_{a 3}=983.21689 \\
& a=5.3023715 \cdot 10^{-3},
\end{aligned}
$$

from these we can write:

$$
g-978.03101 \frac{1+.00193167 \sin ^{2} \varphi}{\left[1-.00669461 \sin ^{2} \varphi\right]^{1 / 2}},
$$

which gives the normal gravity.

According to the closed formulae [2'], [3] and [4], the flattening $f$ and the normal gravity have been calculated with an approximation which is limited only by the approximation with which the parameters are known.

We add in Table I the values of modulus of the gravity vector on the adopted ellipsoid, for $\varphi$ varying from $0^{\circ}$ to $90^{\circ}$, with a pass of $1^{\prime}$. 
'Table I

\begin{tabular}{|c|c|c|c|}
\hline \multicolumn{2}{|c|}{$0^{\prime} 978.03101$} & Jo $0^{\prime}$ & .03258 \\
\hline $1^{\prime}$ & .03101 & $1^{\prime}$ & .03263 \\
\hline $\mathbf{2}^{\prime}$ & .03101 & $2^{\prime}$ & .03269 \\
\hline $3^{\prime}$ & .03101 & $3^{\prime}$ & .03274 \\
\hline $4^{\prime}$ & .03102 & $4^{\prime}$ & .03280 \\
\hline $5^{\prime}$ & .03102 & $5^{\prime}$ & .03285 \\
\hline $6^{\prime}$ & .03102 & $6^{\prime}$ & .03191 \\
\hline $7^{\prime}$ & .03103 & $7^{\prime}$ & .03297 \\
\hline $8^{\prime}$ & .03104 & $8^{\prime}$ & .03303 \\
\hline $9^{\prime}$ & .03104 & $9^{\prime}$ & .03309 \\
\hline $10^{\prime}$ & .03105 & $10^{\prime}$ & .03315 \\
\hline $11^{\prime}$ & .03106 & $11^{\prime}$ & .03321 \\
\hline $12^{\prime}$ & .03107 & $12^{\prime}$ & .03327 \\
\hline $13^{\prime}$ & .03108 & $13^{\prime}$ & .03334 \\
\hline $14^{\prime}$ & .03109 & $14^{\prime}$ & .03340 \\
\hline $15^{\prime}$ & .03111 & $15^{\prime}$ & .03347 \\
\hline $16^{\prime}$ & .03112 & $16^{\prime}$ & .03353 \\
\hline $17^{\prime}$ & .03113 & $17^{\prime}$ & .03360 \\
\hline $18^{\prime}$ & .03115 & $18^{\prime}$ & .03367 \\
\hline $19^{\prime}$ & .03117 & $19^{\prime}$ & .03373 \\
\hline $20^{\prime}$ & .03118 & $20^{\prime}$ & .03380 \\
\hline $21^{\prime}$ & .03120 & $21^{\prime}$ & .03387 \\
\hline $22^{\prime}$ & .03122 & $22^{\prime}$ & .03395 \\
\hline $23^{\prime}$ & .03124 & $23^{\prime}$ & .03402 \\
\hline $24^{\prime}$ & .03126 & $24^{\prime}$ & .03409 \\
\hline $25^{\prime}$ & .03128 & $25^{\prime}$ & .03416 \\
\hline $26^{\prime}$ & .03130 & $26^{\prime}$ & .03424 \\
\hline $27^{\prime}$ & .03133 & $27^{\prime}$ & .03431 \\
\hline $28^{\prime}$ & .03135 & $28^{\prime}$ & .03439 \\
\hline $29^{\prime}$ & .03138 & $29^{\prime}$ & .03447 \\
\hline $30^{\prime}$ & .03140 & $30^{\prime}$ & .03455 \\
\hline $31^{\prime}$ & .03143 & $31^{\prime}$ & .03463 \\
\hline $32^{\prime}$ & .03146 & $32^{\prime}$ & .03471 \\
\hline $33^{\prime}$ & .03148 & $33^{\prime}$ & .03479 \\
\hline $34^{\prime}$ & .03151 & $34^{\prime}$ & .03487 \\
\hline $35^{\prime}$ & .03154 & $3 \overline{5}^{\prime}$ & .03495 \\
\hline $36^{\prime}$ & .03157 & $36^{\prime}$ & .03503 \\
\hline $37^{\prime}$ & .03161 & $37^{\prime}$ & .03512 \\
\hline $38^{\prime}$ & .03164 & $38^{\prime}$ & .03520 \\
\hline $39^{\prime}$ & .03167 & $39^{\prime}$ & .03529 \\
\hline $40^{\prime}$ & .03171 & $10^{\prime}$ & .03538 \\
\hline $41^{\prime}$ & 03174 & $41^{\prime}$ & .03546 \\
\hline $42^{\prime}$ & .03178 & $42^{\prime}$ & .03555 \\
\hline $43^{\prime}$ & .03182 & $43^{\prime}$ & .03564 \\
\hline $44^{\prime}$ & .03185 & $44^{\prime}$ & .03573 \\
\hline $45^{\prime}$ & .03189 & $45^{\prime}$ & .03582 \\
\hline $46^{\prime}$ & .03193 & $46^{\prime}$ & .03592 \\
\hline $47^{\prime}$ & .03197 & $47^{\prime}$ & .03601 \\
\hline $48^{\prime}$ & .03201 & $48^{\prime}$ & .03610 \\
\hline $49^{\prime}$ & .03200 & $49^{\prime}$ & .03620 \\
\hline $50^{\prime}$ & .03210 & $50^{\prime}$ & .03629 \\
\hline $5 l^{\prime}$ & .03214 & $51^{\prime}$ & .03639 \\
\hline $52^{\prime}$ & .03219 & $52^{\prime}$ & .03649 \\
\hline $53^{\prime}$ & .03224 & $53^{\prime}$ & .03658 \\
\hline $54^{\prime}$ & .03228 & $54^{\prime}$ & .03668 \\
\hline $55^{\prime}$ & .03233 & $55^{\prime}$ & .03678 \\
\hline $56^{\prime}$ & .03238 & $56^{\prime}$ & .03688 \\
\hline $57^{\prime}$ & .03243 & $57^{\prime}$ & .03699 \\
\hline $58^{\prime}$ & .03248 & $58^{\prime}$ & .03709 \\
\hline $59^{\prime}$ & .03253 & $59^{\prime}$ & .03719 \\
\hline
\end{tabular}

$20 \quad 0^{\prime} 978.03730$ $1^{\prime} \quad .03740$ $2^{\prime} \quad .03751$

$3^{\prime} \quad .03762$

$4^{\prime} \quad .03772$

$5^{\prime} \quad .03783$

$6^{\prime} \quad .03794$

$7^{\prime} \quad .03805$

$8^{\prime} \quad .03816$

$9^{\prime} \quad 03828$

$10^{\prime} \quad .03839$

$11^{\prime} \quad 03850$

$12^{\prime} \quad 03862$

$13^{\prime} \quad 03873$

$14^{\prime} \quad 03885$

$15^{\prime} \quad 03897$

$16^{\prime} .03908$

$17^{\prime} \quad 03920$

$18^{\prime} \quad 03932$

$19^{\prime} \quad .03944$

$20^{\prime} \quad .03957$

$21^{\prime} \quad .03969$

$22^{\prime} .03981$

$23^{\prime} .03994$

$24^{\prime} \quad .04006$

$25^{\prime} \quad 04019$

$26^{\prime} \quad 04032$

$27^{\prime} \quad 04044$

$28^{\prime} \quad .04057$

$29^{\prime} \quad .04070$

$30^{\prime} \quad .04083$

$31^{\prime} \quad 04096$

$32^{\prime} \quad .04110$

$33^{\prime} \quad 0+123$

$34^{\prime} \quad .04136$

$35^{\prime} \quad 04150$

$36^{\prime} \quad 04163$

$\begin{array}{lll}37^{\prime} & 04177\end{array}$

$38^{\prime} \quad 04191$

$39^{\prime} \quad 04205$

$40^{\prime} \quad .04218$

$41^{\prime} \quad 04232$

$42^{\prime} \quad .04247$

$43^{\prime} \quad .04261$

$44^{\prime} \quad .04275$

$45^{\prime} \quad .04289$

$46^{\prime} \quad .0+304$

$\begin{array}{ll}47^{\prime} & .04318\end{array}$

$48^{\prime} \quad .04333$

$49^{\prime} \quad .04348$

$50^{\prime} \quad .04362$

$51^{\prime} \quad .04377$

$52^{\prime} \quad 04392$

$53^{\prime} \quad .04407$

$54^{\prime} \quad .04+22$

$55^{\prime} \quad .0443 \mathrm{~s}$

$56^{\prime} \quad .04453$

$57^{\prime} \quad .04468$

$58^{\prime} \quad .04484$

$59^{\prime}$ $3^{\circ} \quad 0^{\prime} 978.04515$ $l^{\prime} \quad .04531$

2' 04547

$3^{\prime} \quad .04563$

$4^{\prime} \quad .04579$

$5^{\prime} \quad .04595$

$6^{\prime} \quad .04611$

$7^{\prime} \quad .04627$

$8^{\prime} \quad .04643$

$9^{\prime} \quad 04660$

$10^{\prime} \quad .04676$

$11^{\prime} \quad .04693$

$12^{\prime} \quad 04710$

$13^{\prime} \quad 04726$

$14^{\prime} \quad 04743$

$15^{\prime} \quad .04760$

$16^{\prime} \quad .04777$

$17^{\prime} \quad .04794$

$18^{\prime} \quad .04812$

$19^{\prime} \quad .04829$

$20^{\prime} \quad .04846$

$21^{\prime} \quad .04864$

$22^{\prime} \quad .04881$

$23^{\prime} \quad 04899$

$24^{\prime} \quad 04917$

$25^{\prime} .04935$

$26^{\prime} \quad .04953$

$27^{\prime} \quad .04971$

$28^{\prime} \quad .04989$

$29^{\prime} \quad .05007$

$30^{\prime} \quad .05025$

$31^{\prime} \quad .05043$

$32^{\prime} \quad .05062$

$33^{\prime} \quad 05080$

$\begin{array}{ll}34^{\prime} & .05099\end{array}$

$35^{\prime} \quad 05118$

$36^{\prime} \quad .05136$

$37^{\prime} \quad .05155$

$38^{\prime} \quad .05174$

$39^{\prime} \quad .05193$

$40^{\prime} \quad .05212$

$41^{\prime} \quad .05232$

$42^{\prime} \quad .0525 \mathrm{I}$

$43^{\prime} \quad .05270$

$44^{\prime}$.05290)

$\begin{array}{ll}45^{\prime} & .05309\end{array}$

$46^{\prime} \quad .05329$

$\begin{array}{ll}\mathbf{4}^{\prime} & .05349\end{array}$

$\begin{array}{ll}48^{\prime} & .05369\end{array}$

$49^{\prime} \quad .05388$

$50^{\prime} \quad .05408$

$5 \mathrm{l}^{\prime} \quad .05+29$

$52^{\prime} \quad .05449$

$\begin{array}{lll}53^{\prime} & .05469\end{array}$

$54^{\prime} \quad .05489$

$55^{\prime} .05510$

$56^{\prime} \quad .05530$

$57^{\prime} \quad .05551$

$58^{\prime} \quad .05572$

$59^{\prime} \quad .05592$ 
Table I - (continued)

\begin{tabular}{|c|c|c|c|c|c|c|c|}
\hline \multicolumn{2}{|c|}{$0^{\prime} 978.05613$} & $5^{\circ}$ & $0^{\prime} 978.07023$ & \multicolumn{2}{|c|}{$0^{\prime} 978.08742$} & $7^{\circ}$ & $0^{\prime} 978.10769$ \\
\hline $1^{\prime}$ & .05634 & $1^{\prime}$ & .07049 & $1^{\prime}$ & .08774 & $1^{\prime}$ & .10806 \\
\hline $2^{\prime}$ & 05655 & $2^{\prime}$ & .07075 & $2^{\prime}$ & .08805 & $\mathbf{2}^{\prime}$ & .10842 \\
\hline $3^{r}$ & .05676 & $3^{\prime}$ & .07101 & $3^{\prime}$ & .08836 & $3^{\prime}$ & .10879 \\
\hline $4^{\prime}$ & .05697 & $4^{\prime}$ & .07128 & $t^{\prime}$ & .08868 & $4^{\prime}$ & .10916 \\
\hline $5^{\prime}$ & .05719 & $5^{\prime}$ & .07154 & $5^{\prime}$ & .08900 & $5^{\prime}$ & .10952 \\
\hline $6^{\prime}$ & .05740 & $6^{\prime}$ & .07181 & $6^{\prime}$ & 08931 & $6^{\prime}$ & .10989 \\
\hline $7^{\prime}$ & .05762 & $7^{\prime}$ & .07208 & $7^{\prime}$ & .08963 & $7^{\prime}$ & .11026 \\
\hline $8^{\prime}$ & .05783 & $8^{\prime}$ & .07234 & $8^{\prime}$ & .08995 & $8^{\prime}$ & .11063 \\
\hline $9^{\prime}$ & .05805 & $9^{\prime}$ & .07261 & $9^{\prime}$ & .09027 & $9^{\prime}$ & .11100 \\
\hline $10^{\prime}$ & .05827 & $10^{\prime}$ & .07288 & $10^{\prime}$ & .09059 & $10^{\prime}$ & .11137 \\
\hline $11^{\prime}$ & .05848 & $11^{\prime}$ & .07315 & $11^{\prime}$ & .09091 & $11^{\prime}$ & .11174 \\
\hline $12^{\prime}$ & .05870 & $12^{\prime}$ & .07342 & $12^{\prime}$ & .09123 & $12^{\prime}$ & .11212 \\
\hline $13^{\prime}$ & .05892 & $13^{\prime}$ & .07369 & $13^{\prime}$ & .09155 & $13^{\prime}$ & .11249 \\
\hline $14^{\prime}$ & .05914 & $14^{\prime}$ & .07396 & $14^{\prime}$ & .09188 & $14^{\prime}$ & .11287 \\
\hline $15^{\prime}$ & .05936 & $15^{\prime}$ & 07424 & $15^{\prime}$ & .09220 & $15^{\prime}$ & .11324 \\
\hline $16^{\prime}$ & .05959 & $16^{\prime}$ & .07451 & $16^{\prime}$ & .09253 & $16^{\prime}$ & .11362 \\
\hline $17^{\prime}$ & .05981 & $17^{\prime}$ & .07479 & $17^{\prime}$ & .09286 & $17^{\prime}$ & .11399 \\
\hline $18^{\prime}$ & .06003 & $18^{\prime}$ & .07506 & $18^{\prime}$ & .09318 & $18^{\prime}$ & .11437 \\
\hline $19^{\prime}$ & .06026 & $19^{\prime}$ & .07534 & $19^{\prime}$ & .09351 & $19^{\prime}$ & 11475 \\
\hline $20^{\prime}$ & .06049 & $20^{\prime}$ & .07562 & $20^{\prime}$ & .09384 & $20^{\prime}$ & .11513 \\
\hline $21^{\prime}$ & .06071 & $21^{\prime}$ & .07590 & $21^{\prime}$ & .09417 & $21^{\prime}$ & .11551 \\
\hline $22^{\prime}$ & .06094 & $22^{\prime}$ & .07617 & $22^{\prime}$ & .09450 & $22^{\prime}$ & .11589 \\
\hline $23^{\prime}$ & .06117 & $23^{\prime}$ & .07645 & $23^{\prime}$ & .09483 & $23^{\prime}$ & .11628 \\
\hline $24^{\prime}$ & .06140 & $24^{\prime}$ & .07674 & $24^{\prime}$ & .09516 & $24^{\prime}$ & .11666 \\
\hline $25^{\prime}$ & .06163 & $25^{\prime}$ & .07702 & $25^{\prime}$ & .09550 & $25^{\prime}$ & .11704 \\
\hline $26^{\prime}$ & .06186 & $26^{\prime}$ & .07730 & $26^{\prime}$ & .09583 & $26^{\prime}$ & 11743 \\
\hline $27^{\prime}$ & .06209 & $27^{\prime}$ & .07758 & $27^{\prime}$ & 0.7617 & $27^{\prime}$ & .11781 \\
\hline $28^{\prime}$ & .06232 & $28^{\prime}$ & .07787 & $28^{\prime}$ & .09650 & $28^{\prime}$ & .11820 \\
\hline $29^{\prime}$ & .06256 & $29^{\prime}$ & .07815 & $29^{\prime}$ & .09684 & $29^{\prime}$ & .11859 \\
\hline $30^{\prime}$ & .06279 & $30^{\prime}$ & 07844 & $30^{\prime}$ & .09718 & $30^{\prime}$ & .11898 \\
\hline $31^{\prime}$ & .06303 & $31^{\prime}$ & .07873 & $31^{\prime}$ & .09751 & $31^{\prime}$ & .11937 \\
\hline $32^{\prime}$ & .06326 & $32^{\prime}$ & .07901 & $32^{\prime}$ & .09785 & $32^{\prime}$ & .11976 \\
\hline $33^{\prime}$ & .06350 & $33^{\prime}$ & .07930 & $33^{\prime}$ & .09819 & $33^{\prime}$ & .12015 \\
\hline $34^{\prime}$ & .06374 & $34^{\prime}$ & .07959 & $34^{\prime}$ & .09853 & $3 t^{\prime}$ & .12054 \\
\hline $35^{\prime}$ & .06398 & $35^{\prime}$ & .07988 & $35^{\prime}$ & .09888 & $35^{\prime}$ & .12093 \\
\hline $36^{\prime}$ & .06422 & $36^{\prime}$ & .08017 & $36^{\prime}$ & .09922 & $36^{\prime}$ & .12133 \\
\hline $37^{\prime}$ & .06446 & $37^{\prime}$ & .08047 & $37^{\prime}$ & .09956 & $37^{\prime}$ & .12172 \\
\hline $38^{\prime}$ & .06470 & $38^{\prime}$ & .08076 & $38^{\prime}$ & 09991 & $38^{\prime}$ & .12211 \\
\hline $39^{\prime}$ & .06494 & $39^{\prime}$ & .08105 & $39^{\prime}$ & .10025 & $39^{\prime}$ & .12251 \\
\hline $40^{\prime}$ & .06518 & $40^{\prime}$ & .08135 & $40^{\prime}$ & .10060 & $40^{\prime}$ & .12291 \\
\hline $41^{\prime}$ & .06543 & $4 l^{\prime}$ & .08164 & $41^{\prime}$ & .10094 & $41^{\prime}$ & .12330 \\
\hline $42^{\prime}$ & .06567 & $42^{\prime}$ & .08194 & $42^{\prime}$ & .10129 & $42^{\prime}$ & .12370 \\
\hline $43^{\prime}$ & .06592 & $43^{\prime}$ & .08224 & $43^{\prime}$ & .10164 & $43^{\prime}$ & .12410 \\
\hline $44^{\prime}$ & .06617 & $44^{\prime}$ & .08254 & $44^{\prime}$ & .10199 & $44^{\prime}$ & .12450 \\
\hline $45^{\prime}$ & $.066+1$ & $45^{\prime}$ & .08284 & $45^{\prime}$ & .10234 & $45^{\prime}$ & .12490 \\
\hline $46^{\prime}$ & .066666 & $46^{\prime}$ & .08313 & $46^{\prime}$ & .10269 & $46^{\prime}$ & .12531 \\
\hline $47^{\prime}$ & .06691 & $47^{\prime}$ & 08344 & $47^{\prime}$ & .10304 & $47^{\prime}$ & .12571 \\
\hline $48^{\prime}$ & .06716 & $48^{\prime}$ & .08374 & $48^{\prime}$ & 10340 & $48^{\prime}$ & .12611 \\
\hline $49^{\prime}$ & $.067+1$ & $49^{\prime}$ & .08404 & $49^{\prime}$ & .10375 & $49^{\prime}$ & .12652 \\
\hline $50^{\prime}$ & .06766 & $50^{\prime}$ & .08434 & $50^{\prime}$ & .10410 & $50^{\prime}$ & .12692 \\
\hline $5 l^{\prime}$ & .06792 & $51^{\prime}$ & .08465 & $51^{\prime}$ & .10446 & $51^{\prime}$ & 12733 \\
\hline $52^{\prime}$ & .06817 & $52^{\prime}$ & .08495 & $52^{\prime}$ & 10482 & $52^{\prime}$ & .12773 \\
\hline $53^{\prime}$ & .06842 & $53^{\prime}$ & .08526 & $53^{\prime}$ & .10517 & $53^{\prime}$ & .12814 \\
\hline $5^{d^{\prime}}$ & .06868 & $54^{\prime}$ & .08556 & $54^{\prime}$ & .10553 & $54^{\prime}$ & .12855 \\
\hline $55^{\prime}$ & .06894 & $55^{\prime}$ & .08587 & $55^{\prime}$ & .10589 & $55^{\prime}$ & $.12 \$ 96$ \\
\hline $56^{\prime}$ & .06919 & $56^{\prime}$ & .08618 & $56^{\prime}$ & .10625 & $56^{\prime}$ & .12937 \\
\hline $57^{\prime}$ & .06945 & $57^{\prime}$ & .08649 & $57^{\prime}$ & 10661 & $57^{\prime}$ & .12978 \\
\hline $58^{\prime}$ & .06971 & $58^{\prime}$ & .08680 & $58^{\prime}$ & .10697 & $58^{\prime}$ & .13019 \\
\hline $59^{\prime}$ & .06997 & $59^{\prime}$ & .08711 & $59^{\prime}$ & .10733 & $59^{\prime}$ & .13061 \\
\hline
\end{tabular}


Table I - (continued)

$8^{\circ}$

078.13102

l' .13143

$2^{\prime} \quad .13185$

$3^{\prime} \quad .13227$

$4^{\prime} \quad .13268$

$5^{\prime} \quad .13310$

$6^{\prime} \quad .13352$

$7^{\prime} \quad .13394$

$8^{\prime} \quad .13436$

$9^{\prime} \quad .13478$

$10^{\prime} \quad .13520$

$11^{\prime} .13563$

$12^{\prime} .13605$

$13^{\prime} \quad .13647$

$14^{\prime} .13690$

$15^{\prime} .13732$

$16^{\prime} .13775$

$17^{\prime} \quad .13818$

$18^{\prime} .13861$

$19^{\prime} \quad .13904$

$20^{\prime} \quad .13947$

$21^{\prime} .13990$

$22^{\prime} \quad .14033$

$23^{\prime} \quad .14076$

$24^{\prime} .14120$

$25^{\prime} .14163$

$26^{\prime} .14207$

$27^{\prime} \quad .14250$

$28^{\prime} \quad .14294$

$29^{\prime} \quad .14338$

$30^{\prime} \quad .14382$

$3 I^{\prime} \quad .14426$

$32^{\prime} \quad 14470$

$33^{\prime} \quad .14514$

$34^{\prime} \quad 14558$

$35^{\prime} \quad .14602$

$36^{\prime} \quad .14647$

$37^{\prime} \quad .14691$

$38^{\prime} \quad .14736$

$39^{\prime} \quad .14780$

$40^{\prime} .14825$

$41^{\prime} .14870$

$42^{\prime} .14915$

$43^{\prime} \quad .14960$

$44^{\prime} .15005$

$45^{\prime} \quad .15050$

$46^{\prime} \quad .15095$

$47^{\prime} .15141$

$48^{\prime} \quad .15186$

$49^{\prime} \quad .15231$

$50^{\prime} \quad .15277$

$51^{\prime} .15323$

$52^{\prime} \quad .15368$

$53^{\prime} \quad .15414$

$54^{\prime} \quad .15460$

$55^{\prime} \quad .15506$

$56^{\prime} \quad .15552$

$57^{\prime} \quad .15598$

$58^{\prime} \quad .15644$ $59^{\prime}$ $9^{\circ} \quad 0^{\prime} 978.15737$

$$
1^{\prime} \quad .15783
$$

$2^{\prime} \quad .15830$

$3^{\prime} \quad .15877$

$4^{\prime} .15923$

$5^{\prime} \quad .15970$

$6^{\prime} \quad .16017$

$7^{\prime} \quad .16064$

$8^{\prime} .16111$

$9^{\prime} \quad .16158$

$10^{\prime} \quad .16205$

] $1^{\prime} .16253$

$12^{\prime} .16300$

$13^{\prime} \quad .16347$

$14^{\prime} .16395$

$15^{\prime} .16443$

$16^{\prime} .16490$

$17^{\prime} .16538$

$18^{\prime} .16586$

$19^{\prime} \quad .16634$

$20^{\prime} \quad .16682$

$2 \mathrm{I}^{\prime} \quad .16730$

$22^{\prime} \quad .16778$

$23^{\prime} \quad 16827$

$24^{\prime} .16875$

$25^{\prime} \quad .16923$

$26^{\prime} \quad .16972$

$27^{\prime} .17021$

$28^{\prime} \quad .17069$

$29^{\prime} \quad .17118$

$30^{\prime} \quad .17167$

$3 I^{\prime} \quad 17216$

$\begin{array}{ll}32^{\prime} & .17265\end{array}$

$33^{\prime} \quad .17314$

$34^{\prime} \quad 17363$

$35^{\prime} \quad .17412$

$36^{\prime} \quad .17462$

$37^{\prime} .17511$

$38^{\prime} .1756 \mathrm{I}$

$39^{\prime} \quad .17610$

$40^{\prime} \quad .17660$

$41, \quad 17710$

$42^{\prime} \quad .17760$

$43^{\prime} \quad .17810$

$44^{\prime} \quad .17860$

$45^{\prime} \quad .17910$

$46^{\prime} \quad .17960$

$47^{\prime} \quad .18010$

$48^{\prime} \quad .18061$

$49^{\prime} \quad .18111$

$50^{\prime} \quad .18162$

$51^{\prime} .18212$

$52^{\prime} \quad .18263$

$53^{\prime} \quad .18314$

$54^{\prime} .18364$

$55^{\prime} .18415$

$56^{\prime} \quad .18466$

$57^{\prime} \quad .18517$

$58^{\prime} \quad .18569$

$59^{\prime} \quad .18620$ $10^{\circ} \quad 0^{\prime} 978.18671$

$\begin{array}{ll}\mathrm{I}^{\prime} & .18723 \\ 2^{\prime} & .18774 \\ 3^{\prime} & .18826 \\ 4^{\prime} & .18877 \\ 5^{\prime} & .18929 \\ 6^{\prime} & .18981 \\ 7^{\prime} & .19033 \\ 8^{\prime} & .19085 \\ 9^{\prime} & .19137\end{array}$

$10^{\prime} \quad .19189$

$11^{\prime} .19241$

$12^{\prime} \quad .19294$

$13^{\prime} \quad .19346$

$14^{\prime} \quad .19399$

$15^{\prime} .19451$

$16^{\prime} .19504$

$17^{\prime} \quad .19557$

$18^{\prime} .19609$

$19^{\prime} \quad .19662$

$20^{\prime} \quad .19715$

$21^{\prime} \quad .19768$

$22^{\prime} \quad .19821$

$23^{\prime} \quad 19875$

$24^{\prime} \quad .19928$

$25^{\prime} \quad .1998$ I

$26^{\prime} \quad .20035$

$27^{\prime} \quad .20088$

$28^{\prime} \quad .20142$

$29^{\prime} \quad .20196$

$30^{\prime} \quad .20250$

$31^{\prime} \quad .20303$

$32^{\prime} \quad .20357$

$33^{\prime} \quad .20411$

$34^{\prime} \quad 20466$

$35^{\prime} \quad .20520$

$36^{\prime}$

$37^{\prime}$

$38^{\prime}$

$39^{\prime}$

$40^{\prime}$

$4 \mathrm{l}^{\prime}$

$42^{\prime}$

$43^{\prime}$

$44^{\prime}$

$45^{\prime}$

$46^{\prime}$

$47^{\prime}$

$48^{\prime}$

$49^{\prime}$

$50^{\prime}$

$51^{\prime}$

$52^{\prime}$

$53^{\prime}$

$54^{\prime}$

$55^{\prime}$

$56^{\prime}$

$57^{\prime}$

$58^{\prime}$

$59^{\prime}$ $11^{\circ} \quad 0^{\prime} 978.21901$

$l^{\prime}-21958$

$2^{\prime} \quad 22014$

$3^{\prime} \quad .22071$

$4^{\prime} \quad .22127$

$5^{\prime} \quad .22184$

$6^{\prime} \quad .22240$

$\begin{array}{ll}7^{\prime} & 22297\end{array}$

$8^{\prime} \quad .22354$

$9^{\prime} \quad .22411$

$10^{\prime} \quad .22468$

$11^{\prime} .22525$

$12^{\prime} \quad .22583$

$13^{\prime} \quad .22640$

$14^{\prime} \quad .22697$

$15^{\prime} \quad .22755$

$16^{\prime} .22812$

$17^{\prime} \quad .22870$

$18^{\prime} \quad .22927$

$19^{\prime} \quad .22985$

$20^{\prime} \quad .23043$

$21^{\prime} .23101$

$22^{\prime} \quad .23159$

$23^{\prime} \quad .23217$

$24^{\prime} \quad .23275$

$25^{\prime} \quad .23334$

$26^{\prime} \quad 23392$

$27^{\prime} \quad .23450$

$28^{\prime} \quad .23509$

$29^{\prime} \quad .23567$

$30^{\prime} \quad .23626$

$31^{\prime} \quad .23685$

$32^{\prime} \quad .23744$

$33^{\prime} \quad .23803$

34' .23862

$35^{\prime} \quad .23921$

$36^{\prime} \quad .23980$

$37^{\prime} \quad .24039$

$38^{\prime} \quad .24098$

$39^{\prime} \quad .24158$

$40^{\prime} \quad .24217$

$41^{\prime} \quad .24277$

$42^{\prime} \quad .24336$

$43^{\prime} \quad .24396$

$44^{\prime} \quad .24456$

$45^{\prime} \quad .24516$

$46^{\prime} \quad .24576$

$47^{\prime} \quad .24636$

$48^{\prime} \quad .24696$

$49^{\prime} \quad .24756$

$50^{\prime} \quad .24816$

$51^{\prime} .24877$

$52^{\prime} \quad .24937$

$53^{\prime} \quad .24998$

$54^{\prime} \quad .25058$

$55^{\prime} \quad .25119$

$56^{\prime} \quad .25180$

$57^{\prime} \quad .25240$

$58^{\prime} \quad .25301$

$59^{\prime} \quad .25362$ 
'Table I -. (continued)

\begin{tabular}{|c|c|c|c|c|c|c|c|}
\hline $0^{\prime} 9$ & .25423 & $13^{\circ}$ & 3.29233 & $14^{\circ}$ & 3.33326 & $15^{\circ}$ & $0^{\prime} 978.37697$ \\
\hline $1^{\prime}$ & .25484 & $l^{\prime}$ & .29299 & $1^{\prime}$ & .33396 & & $1^{\prime} \quad .37772$ \\
\hline $2^{\prime}$ & .25546 & $2^{\prime}$ & .29365 & $2^{\prime}$ & .33467 & & .37847 \\
\hline $3^{\prime}$ & .25607 & $3^{\prime}$ & .29431 & $3^{\prime}$ & .33538 & & .37922 \\
\hline $4^{\prime}$ & .25668 & $4^{\prime}$ & .29497 & $4^{\prime}$ & .33609 & & .37998 \\
\hline $5^{\prime}$ & .25730 & $5^{\prime}$ & .29563 & $5^{\prime}$ & .33679 & & .38073 \\
\hline $6^{\prime}$ & .25791 & $6^{\prime}$ & .29630 & $6^{\prime}$ & .33750 & & .38149 \\
\hline $7^{\prime}$ & .25853 & $7^{\prime}$ & .29696 & $7^{\prime}$ & .33821 & & .38224 \\
\hline $8^{\prime}$ & .25915 & $8^{\prime}$ & .29762 & $8^{\prime}$ & .33893 & & .38300 \\
\hline $9^{\prime}$ & .25977 & $9^{\prime}$ & .29829 & $9^{\prime}$ & .33964 & & .38376 \\
\hline $10^{\prime}$ & .26038 & $10^{\prime}$ & .29896 & $10^{\prime}$ & 34035 & & .38452 \\
\hline $11^{\prime}$ & .26100 & $11^{\prime}$ & .29962 & $11^{\prime}$ & .34106 & & .38528 \\
\hline $12^{\prime}$ & 26162 & $12^{\prime}$ & .30029 & $12^{\prime}$ & 34178 & & .38604 \\
\hline $13^{\prime}$ & .26224 & $13^{\prime}$ & .30096 & $13^{\prime}$ & 34249 & & .38680 \\
\hline $14^{\prime}$ & .26287 & $14^{\prime}$ & .30163 & $14^{\prime}$ & .34321 & & .38756 \\
\hline $15^{\prime}$ & .26349 & $15^{\prime}$ & .30230 & $15^{\prime}$ & .34393 & & .38832 \\
\hline $16^{\prime}$ & .26411 & $16^{\prime}$ & .30297 & $16^{\prime}$ & .34464 & & .38908 \\
\hline $17^{\prime}$ & .26474 & $17^{\prime}$ & .30364 & $17^{\prime}$ & .34536 & & .38985 \\
\hline $18^{\prime}$ & .26536 & $18^{\prime}$ & .30431 & $18^{\prime}$ & .34608 & & .39061 \\
\hline $19^{\prime}$ & .26599 & $19^{\prime}$ & .30499 & $19^{\prime}$ & .34680 & & .39138 \\
\hline $20^{\prime}$ & $.2666 \mathrm{I}$ & $20^{\prime}$ & .30566 & $20^{\prime}$ & .34752 & & .39214 \\
\hline $21^{\prime}$ & .26724 & $21^{\prime}$ & .30633 & $21^{\prime}$ & .34824 & & .39291 \\
\hline $22^{\prime}$ & .26787 & $22^{\prime}$ & .30701 & $22^{\prime}$ & .34896 & & .39368 \\
\hline $23^{\prime}$ & .26850 & $23^{\prime}$ & .30769 & $23^{\prime}$ & .34969 & & .39445 \\
\hline $24^{\prime}$ & .26913 & $24^{\prime}$ & .30836 & $24^{\prime}$ & .35041 & & .39522 \\
\hline $25^{\prime}$ & .26976 & $25^{\prime}$ & .30904 & $25^{\prime}$ & .35113 & & .39599 \\
\hline $26^{\prime}$ & .27039 & $26^{\prime}$ & .30972 & $26^{\prime}$ & .35186 & & .39676 \\
\hline $27^{\prime}$ & .27102 & $27^{\prime}$ & .31040 & $27^{\prime}$ & .35258 & & .39753 \\
\hline $28^{\prime}$ & .27166 & $28^{\prime}$ & .31108 & $28^{\prime}$ & .35331 & & .39830 \\
\hline $29^{\prime}$ & .27229 & $29^{\prime}$ & .31176 & $29^{\prime}$ & .35404 & & .39907 \\
\hline $30^{\prime}$ & .27292 & $30^{\prime}$ & .31244 & $30^{\prime}$ & .35477 & & .39985 \\
\hline $31^{\prime}$ & .27356 & $31^{\prime}$ & .31313 & $3 \mathrm{I}^{\prime}$ & .35550 & & .40062 \\
\hline $32^{\prime}$ & .27420 & $32^{\prime}$ & .31381 & $32^{\prime}$ & .35623 & & .40140 \\
\hline $33^{\prime}$ & .27483 & $33^{\prime}$ & .31449 & $33^{\prime}$ & .35696 & & .40217 \\
\hline $34^{\prime}$ & .27547 & $34^{\prime}$ & .31518 & $34^{\prime}$ & .35769 & & .40295 \\
\hline $35^{\prime}$ & .27611 & $35^{\prime}$ & .31586 & $35^{\prime}$ & .35842 & & .40373 \\
\hline $36^{\prime}$ & .27675 & $36^{\prime}$ & .31655 & $36^{\prime}$ & .35915 & & .40450 \\
\hline $37^{\prime}$ & .27739 & $37^{\prime}$ & 31724 & $37^{\prime}$ & .35989 & & .40528 \\
\hline $38^{\prime}$ & .27803 & $38^{\prime}$ & 31792 & $38^{\prime}$ & .36062 & & .40606 \\
\hline $39^{\prime}$ & .27867 & $39^{\prime}$ & .31861 & $39^{\prime}$ & .36135 & & .40684 \\
\hline $40^{\prime}$ & .27931 & $40^{\prime}$ & .31930 & $40^{\prime}$ & .36209 & & .40762 \\
\hline $41^{\prime}$ & .27996 & $41^{\prime}$ & .31999 & $41^{\prime}$ & .36283 & & .40841 \\
\hline $42^{\prime}$ & .28060 & $42^{\prime}$ & .32068 & $42^{\prime}$ & .36356 & & .40919 \\
\hline $43^{\prime}$ & .28125 & $43^{\prime}$ & .32138 & $43^{\prime}$ & .36430 & & .40997 \\
\hline $44^{\prime}$ & .28189 & $44^{\prime}$ & .32207 & $44^{\prime}$ & .36504 & & .41076 \\
\hline $45^{\prime}$ & .28254 & $45^{\prime}$ & .32276 & $45^{\prime}$ & .36578 & & .41154 \\
\hline $46^{\prime}$ & .28319 & $46^{\prime}$ & .32346 & $46^{\prime}$ & .36652 & & .41233 \\
\hline $47^{\prime}$ & .28383 & $47^{\prime}$ & $.32+15$ & $47^{\prime}$ & .36726 & & .41311 \\
\hline $48^{\prime}$ & .28448 & $48^{\prime}$ & .32485 & $48^{\prime}$ & .36800 & & .41390 \\
\hline $49^{\prime}$ & .28513 & $49^{\prime}$ & .32554 & $49^{\prime}$ & .368 & & .41469 \\
\hline $50^{\prime}$ & .28578 & $50^{\prime}$ & .32624 & $50^{\prime}$ & .36949 & & .41548 \\
\hline $5 l^{\prime}$ & .28643 & $51^{\prime}$ & .32694 & $51^{\prime}$ & .37023 & & .41627 \\
\hline $52^{\prime}$ & .28709 & $52^{\prime}$ & .32764 & $52^{\prime}$ & .37098 & & .41706 \\
\hline $53^{\prime}$ & .28774 & $53^{\prime}$ & .32834 & $53^{\prime}$ & .37172 & & .41785 \\
\hline $54^{\prime}$ & .28839 & $54^{\prime}$ & .32904 & $54^{\prime}$ & .37247 & & .41864 \\
\hline $55^{\prime}$ & .28905 & $55^{\prime}$ & .32974 & $55^{\prime}$ & .37322 & & .41943 \\
\hline $56^{\prime}$ & .28970 & $56^{\prime}$ & .33044 & $56^{\prime}$ & .37397 & & .42022 \\
\hline $57^{\prime}$ & .29036 & $57^{\prime}$ & .33114 & $57^{\prime}$ & .37472 & & .42102 \\
\hline $58^{\prime}$ & .29101 & $58^{\prime}$ & .33185 & $58^{\prime}$ & .37546 & & .42181 \\
\hline $59^{\prime}$ & .29167 & $59^{\prime}$ & .33255 & $59^{\prime}$ & .37621 & & .42261 \\
\hline
\end{tabular}


Table I - (continued)

\begin{tabular}{|c|c|c|c|c|c|c|c|}
\hline $0^{\prime}$ & 3.42340 & $17^{\circ}$ & 3.47251 & $18^{\circ}$ & 3.52424 & $19^{\circ}$ & 8.57851 \\
\hline$I^{\prime}$ & .42420 & $\mathrm{l}^{\prime}$ & .47335 & $1^{\prime}$ & .52512 & $1^{\prime}$ & .57944 \\
\hline $2^{\prime}$ & .42500 & $\underline{2}^{\prime}$ & .47420 & $2^{\prime}$ & .52601 & $2^{\prime}$ & .58037 \\
\hline $3^{\prime}$ & .42580 & $3^{\prime}$ & .47504 & $3^{\prime}$ & .52689 & $3^{\prime}$ & .58129 \\
\hline $4^{\prime}$ &.+2660 & $4^{\prime}$ & .47588 & $4^{\prime}$ & .52778 & $4^{\prime}$ & .5822 .2 \\
\hline $5^{\prime}$ & .42739 & $5^{\prime}$ & .47673 & $5^{\prime}$ & .52866 & $5^{\prime}$ & .58315 \\
\hline $6^{\prime}$ & .42820 & $6^{\prime}$ & .47757 & $6^{\prime}$ & .52955 & $6^{\prime}$ & .58408 \\
\hline $7^{\prime}$ & .42900 & $\tau^{\prime}$ & .47842 & $7^{\prime}$ & .53044 & $7^{\prime}$ & .58501 \\
\hline $8^{\prime}$ & .42980 & $8^{\prime}$ & .47926 & $8^{\prime}$ & .53133 & $8^{\prime}$ & .58594 \\
\hline $9^{\prime}$ & .43060 & $9^{\prime}$ & .48011 & $9^{\prime}$ & .53222 & $9^{\prime}$ & .58687 \\
\hline $10^{\prime}$ & .43140 & $10^{\prime}$ & .48096 & $10^{\prime}$ & .53311 & $10^{\prime}$ & .58780 \\
\hline $11^{\prime}$ & .43221 & $11^{\prime}$ & .48180 & $1 \mathrm{I}^{\prime}$ & .53400 & $11^{\prime}$ & .58874 \\
\hline $12^{\prime}$ & .43301 & $12^{\prime}$ & .48265 & $12^{\prime}$ & .53489 & $12^{\prime}$ & .58967 \\
\hline $13^{\prime}$ & .43382 & $13^{\prime}$ & .48350 & $13^{\prime}$ & 53578 & $13^{\prime}$ & .59060 \\
\hline $14^{\prime}$ & .43463 & $14^{\prime}$ & .48435 & $14^{\prime}$ & .53668 & $14^{\prime}$ & .59154 \\
\hline $15^{\prime}$ & .43543 & $15^{\prime}$ & .48520 & $15^{\prime}$ & .53757 & $15^{\prime}$ & .59247 \\
\hline $16^{\prime}$ & .43624 & $16^{\prime}$ & .48605 & $16^{\prime}$ & .53847 & $16^{\prime}$ & $.593+1$ \\
\hline $17^{\prime}$ & .43705 & $17^{\prime}$ & .48691 & $17^{\prime}$ & .53936 & $17^{\prime}$ & .59435 \\
\hline $18^{\prime}$ & .43786 & $18^{\prime}$ & .48776 & $18^{\prime}$ & .54026 & $18^{\prime}$ & .59528 \\
\hline $19^{\prime}$ & .43867 & $19^{\prime}$ & .48861 & $19^{\prime}$ & .54115 & $19^{\prime}$ & .59622 \\
\hline 2()$^{\prime}$ & .43948 & 2()$^{\prime}$ & .48047 & $20^{\prime}$ & .54205 & $20^{\prime}$ & .59716 \\
\hline $21^{\prime}$ & .44029 & $21^{\prime}$ & .49032 & $21^{\prime}$ & .54295 & $21^{\prime}$ & .59810 \\
\hline $22^{\prime}$ & .44110 & $22^{\prime}$ & .49118 & $22^{\prime}$ & .54385 & $22^{\prime}$ & .59904 \\
\hline $23^{\prime}$ & .44192 & $23^{\prime}$ & .49204 & $23^{\prime}$ & .54475 & $23^{\prime}$ & .59998 \\
\hline $24^{\prime}$ & .44273 & $24^{\prime}$ & .49289 & $24^{\prime}$ & .54565 & $24^{\prime}$ & .60092 \\
\hline $25^{\prime}$ & .44354 & $255^{\prime}$ & .49375 & $25^{\prime}$ & .54655 & $25^{\prime}$ & .60187 \\
\hline $26^{\prime}$ & .44436 & $26^{\prime}$ & .49461 & $26^{\prime}$ & .54745 & $26^{\prime}$ & .60281 \\
\hline $27^{\prime}$ & .44518 & $27^{\prime}$ & .49547 & $27^{\prime}$ & .54835 & $27^{\prime}$ & .60375 \\
\hline $28^{\prime}$ & .44599 & $2 s^{\prime}$ & .49633 & $28^{\prime}$ & .54925 & $28^{\prime}$ & .60470 \\
\hline $29^{\prime}$ & .44681 & $29^{\prime}$ & .49719 & $29^{\prime}$ & .55016 & $29^{\prime}$ & .60564 \\
\hline $30^{\prime}$ & .44763 & $30^{\prime}$ & .49805 & $30^{\prime}$ & .55106 & $30^{\prime}$ & .60659 \\
\hline $31^{\prime}$ & .44845 & $31^{\prime}$ & .49892 & $31^{\prime}$ & .55197 & $31^{\prime}$ & .60754 \\
\hline $32^{\prime}$ & .44927 & $32^{\prime}$ & .49978 & $32^{\prime}$ & .55287 & $32^{\prime}$ & .60848 \\
\hline $33^{\prime}$ & .45009 & $33^{\prime}$ & .50064 & $33^{\prime}$ & .55378 & $33^{\prime}$ & .60943 \\
\hline $34^{\prime}$ & .45091 & $34^{\prime}$ & .50151 & $34^{\prime}$ & .55469 & $34^{\prime}$ & .61038 \\
\hline $34^{\prime}$ & .45173 & $35^{\prime}$ & .50237 & $35^{\prime}$ & .55559 & $35^{\prime}$ & .61133 \\
\hline $35^{\prime}$ & .45255 & $36^{\prime}$ & .50324 & $36^{\prime}$ & .55650 & $36^{\prime}$ & .61228 \\
\hline $37^{\prime}$ & .45338 & $37^{\prime}$ & $.50+11$ & $37^{\prime}$ & .55741 & $37^{\prime}$ & .61323 \\
\hline $38^{\prime}$ & .45420 & $38^{\prime}$ & .50497 & $38^{\prime}$ & .55832 & $38^{\prime}$ & .61418 \\
\hline $39^{\prime}$ & .45503 & $39^{\prime}$ & .50584 & $39^{\prime}$ & .55923 & $39^{\prime}$ & .61513 \\
\hline $40^{\prime}$ & .45585 & $40^{\prime}$ & .50671 & $40)^{\prime}$ & .56014 & $40^{\prime}$ & .61608 \\
\hline $41^{\prime}$ & .45668 & $41^{\prime}$ & .50758 & $41^{\prime}$ & .56105 & $4 I^{\prime}$ & .61704 \\
\hline $42^{\prime}$ & .45750 & $42^{\prime}$ & .50845 & $42^{\prime}$ & .56197 & $42^{\prime}$ & .61799 \\
\hline $43^{\prime}$ & .45833 & $43^{\prime}$ & .50932 & $43^{\prime}$ & .56288 & $43^{\prime}$ & .61895 \\
\hline $44^{\prime}$ & .45916 & $44^{\prime}$ & .51019 & $44^{\prime}$ & .56379 & $44^{\prime}$ & .61990 \\
\hline $45^{\prime}$ & .45999 & $45^{\prime}$ & & $45^{\prime}$ & .56471 & $45^{\prime}$ & .62086 \\
\hline $46^{\prime}$ & .460182 & $46^{\prime}$ & .51194 & $46^{\prime}$ & .56563 & $46^{\prime}$ & .62181 \\
\hline $47^{\prime}$ & .46165 & $47^{\prime}$ & .51281 & $47^{\prime}$ & .566554 & $47^{\prime}$ & .62277 \\
\hline $48^{\prime}$ & 46249 & $48^{\prime}$ & .51369 & $48^{\prime}$ & .56746 & $48^{\prime}$ & .62373 \\
\hline $49^{\prime}$ & .46331 & $49^{\prime}$ & .51456 & $49^{\prime}$ & .56838 & $49^{\prime}$ & .62469 \\
\hline 50$)^{\prime}$ & $46+15$ & $50^{\prime}$ & .51544 & $50^{\prime}$ & .56929 & $50^{\prime}$ & .62564 \\
\hline $5 I^{\prime}$ & .46498 & $5 l^{\prime}$ & .51632 & $5 l^{\prime}$ & .57021 & $5 l^{\prime}$ & .62661 \\
\hline $52^{\prime}$ & .46581 & $52^{\prime}$ & .51719 & $52^{\prime}$ & .57113 & $52^{\prime}$ & .62757 \\
\hline $53^{\prime}$ & 46665 & $53^{\prime}$ & .51807 & $53^{\prime}$ & .57205 & $53^{\prime}$ & .62853 \\
\hline $54^{\prime}$ & 46748 & $54^{\prime}$ & .51895 & $54^{\prime}$ & .57297 & $54^{\prime}$ & .62949 \\
\hline $55^{\prime}$ & 46832 & $55^{\prime}$ & .51983 & $55^{\prime}$ & .57390 & $55^{\prime}$ & .63045 \\
\hline $56^{\prime}$ & 46916 & $56^{\prime}$ & .52071 & $56^{\prime}$ & .57482 & $56^{\prime}$ & .63142 \\
\hline $57^{\prime}$ & 47000 & $57^{\prime}$ & .52159 & $57^{\prime}$ & .57574 & $57^{\prime}$ & .63238 \\
\hline $58^{\prime}$ & 47083 & $58^{\prime}$ & .52247 & $58^{\prime}$ & .57666 & $58^{\prime}$ & .63334 \\
\hline $59^{\prime}$ & 47167 & $59^{\prime}$ & .52336 & $59^{\prime}$ & .57759 & $59^{\prime}$ & .63431 \\
\hline
\end{tabular}


Table I - (continued)

\begin{tabular}{|c|c|c|c|c|c|c|c|}
\hline$\sigma^{\prime}$ & 8.63528 & $21^{\circ}$ & $0^{\prime} 978.69446$ & $22^{\circ}$ & ()$^{\prime} 978.75598$ & $23^{\circ}$ & 8.81978 \\
\hline $1^{\prime}$ & .63624 & & $I^{\prime} \quad .69546$ & & $l^{\prime} \quad .75703$ & $\mathbf{I}^{\prime}$ & .82086 \\
\hline $2^{\prime}$ & .63721 & & .69647 & & .75807 & $2^{\prime}$ & .82194 \\
\hline $3^{\prime}$ & .63818 & & .69748 & & .75912 & $\overline{3}^{\prime}$ & .82303 \\
\hline $4^{\prime}$ & .63915 & & $.698+8$ & & .76016 & $4^{\prime}$ & .82411 \\
\hline 5$)^{\prime}$ & .64012 & & 69949 & & .76121 & $5^{\prime}$ & .82520 \\
\hline $6^{\prime}$ & .64109 & & .70050 & & .76226 & $6^{\prime}$ & .82628 \\
\hline $7^{\prime}$ & .64206 & & .70151 & & .76331 & $7^{\prime}$ & .82737 \\
\hline $8^{\prime}$ & .64303 & & .70252 & & .76436 & $8^{\prime}$ & .82845 \\
\hline $9^{\prime}$ & .64400 & & .70354 & & $.765+1$ & $9^{\prime}$ & .82954 \\
\hline $10^{\prime}$ & .64497 & & .70455 & $10^{\prime}$ & .76646 & $10^{\prime}$ & .83063 \\
\hline $\mathrm{II}^{\prime}$ & .64595 & & .70556 & $11^{\prime}$ & .76751 & $11^{\prime}$ & .83172 \\
\hline $12^{\prime}$ & .64692 & & .70658 & $12^{\prime}$ & .76856 & $12^{\prime}$ & .83280 \\
\hline $13^{\prime}$ & .64790 & & .70759 & $13^{\prime}$ & .76961 & $13^{\prime}$ & .83389 \\
\hline $14^{\prime}$ & .64887 & & .70860 & $14^{\prime}$ & .77067 & $14^{\prime}$ & .83498 \\
\hline $15^{\prime}$ & .64985 & & .70962 & $15^{\prime}$ & .77172 & $15^{\prime}$ & .83607 \\
\hline $16^{\prime}$ & .65082 & & .71064 & $16^{\prime}$ & .77277 & $16^{\prime}$ & .83717 \\
\hline $17^{\prime}$ & .65180 & & .71165 & $17^{\prime}$ & .77383 & $17^{\prime}$ & .83826 \\
\hline $18^{\prime}$ & .65278 & & .71267 & $18^{\prime}$ & .77489 & $18^{\prime}$ & .83935 \\
\hline $19^{\prime}$ & .65376 & & .71369 & $19^{\prime}$ & .77594 & $19^{\prime}$ & .84044 \\
\hline $20^{\prime}$ & .65474 & & $.71+71$ & $20^{\prime}$ & .77700 & $20^{\prime}$ & .84154 \\
\hline $21^{\prime}$ & 65572 & & .71573 & $21^{\prime}$ & .77806 & $21^{\prime}$ & .84263 \\
\hline $22^{\prime}$ & .65670 & & .71675 & $22^{\prime}$ & .77911 & $22^{\prime}$ & .84373 \\
\hline $23^{\prime}$ & .65768 & & .71777 & $23^{\prime}$ & .78017 & $23^{\prime}$ & .84482 \\
\hline $24^{\prime}$ & .65866 & & .71879 & $24^{\prime}$ & 78123 & $24^{\prime}$ & .84592 \\
\hline $25^{\prime}$ & .659964 & & .71981 & $25^{\prime}$ & 78229 & $25^{\prime}$ & .84701 \\
\hline $26^{\prime}$ & .66063 & & .72083 & $26^{\prime}$ & .78335 & $26^{\prime}$ & .84811 \\
\hline $27^{\prime}$ & .66161 & & .72186 & $27^{\prime}$ & .78441 & $27^{\prime}$ & .84921 \\
\hline $28^{\prime}$ & .66260 & & .72288 & $28^{\prime}$ & .78548 & $28^{\prime}$ & .85031 \\
\hline $29^{\prime}$ & 66358 & & .72390 & $29^{\prime}$ & .78654 & $29^{\prime}$ & .85141 \\
\hline $30^{\prime}$ & .66457 & & .72493 & $30^{\prime}$ & .78760 & $30^{\prime}$ & .85251 \\
\hline $31^{\prime}$ & .66555 & & .72596 & $31^{\prime}$ & .78866 & $31^{\prime}$ & .85361 \\
\hline $32^{\prime}$ & .66654 & & .72698 & $32^{\prime}$ & .78973 & $32^{\prime}$ & .85471 \\
\hline $33^{\prime}$ & .66753 & & .71801 & $33^{\prime}$ & .79079 & $33^{\prime}$ & .85581 \\
\hline $34^{\prime}$ & .66852 & & .72904 & $34^{\prime}$ & .79186 & $34^{\prime}$ & .856991 \\
\hline $35^{\prime}$ & .66951 & & .73006 & $35^{\prime}$ & .79292 & $35^{\prime}$ & .85801 \\
\hline $36^{\prime}$ & .67050 & & .73109 & $36^{\prime}$ & .79399 & $36^{\prime}$ & .85912 \\
\hline $37^{\prime}$ & .67149 & & .73212 & $37^{\prime}$ & .79506 & $37^{\prime}$ & .86022 \\
\hline $38^{\prime}$ & .67248 & & .73315 & $38^{\prime}$ & .79613 & $38^{\prime}$ & .86132 \\
\hline $39^{\prime}$ & .67347 & & .73418 & $39^{\prime}$ & .797 & $39^{\prime}$ & .86243 \\
\hline 4$)^{\prime}$ & .67446 & & .73522 & $4)^{\prime}$ & .79826 & $40^{\prime}$ & .86353 \\
\hline $41^{\prime}$ & .67546 & & .73625 & $4 l^{\prime}$ & .79 & $+1^{\prime}$ & .86464 \\
\hline $42^{\prime}$ & .67645 & & .73728 & $42^{\prime}$ & .800 .41 & $42^{\prime}$ & .86575 \\
\hline $43^{\prime}$ & .67745 & & .73 & $43^{\prime}$ & .80 & $43^{\prime}$ & .86685 \\
\hline $44^{\prime}$ & .67844 & & .73935 & $44^{\prime}$ & .80255 & $44^{\prime}$ & .86796 \\
\hline $45^{\prime}$ & .67944 & & .74 & $45^{\prime}$ & 80 & $45^{\prime}$ & .86907 \\
\hline $46^{\prime}$ & .68043 & & .74142 & $46^{\prime}$ & .80469 & $46^{\prime}$ & .87018 \\
\hline $47^{\prime}$ & .68143 & 4 & .742 & $47^{\prime}$ & .80577 & $47^{\prime}$ & .87129 \\
\hline $48^{\prime}$ & .68243 & & .74349 & $48^{\prime}$ & .80684 & $48^{\prime}$ & .87240 \\
\hline $49^{\prime}$ & .68343 & & .74453 & $49^{\prime}$ & .80792 & $49^{\prime}$ & .87351 \\
\hline $50^{\prime}$ & .68443 & & .74557 & $50^{\prime}$ & .80899 & $50)^{\prime}$ & .87462 \\
\hline $5 \mathrm{l}^{\prime}$ & .68543 & & .74661 & $5 l^{\prime}$ & .81007 & $5 \mathrm{l}^{\prime}$ & .87574 \\
\hline $52^{\prime}$ & .68643 & & .74764 & $52^{\prime}$ & .81114 & $52^{\prime}$ & .87685 \\
\hline $53^{\prime}$ & .68743 & & .74868 & $53^{\prime}$ & .81222 & $53^{\prime}$ & .87796 \\
\hline $54^{\prime}$ & .68843 & & .74972 & $54^{\prime}$ & .81330 & $54^{\prime}$ & .87908 \\
\hline $55^{\prime}$ & .68943 & & .75077 & $55^{\prime}$ & $.81+38$ & $55^{\prime}$ & .88019 \\
\hline $56^{\prime}$ & .69044 & & .75181 & $56^{\prime}$ & .81546 & $56^{\prime}$ & .88130 \\
\hline $57^{\prime}$ & $.691+4$ & 5 & $.7528 \pi$ & $57^{\prime}$ & .81654 & $57^{\prime}$ & .88242 \\
\hline $58^{\prime}$ & .69244 & 58 & .75389 & $58^{\prime}$ & .81762 & $58^{\prime}$ & .88354 \\
\hline $59^{\prime}$ & .69345 & & .75494 & $59^{\prime}$ & .81870 & $59^{\prime}$ & .88465 \\
\hline
\end{tabular}


Table I - (continued)

\begin{tabular}{|c|c|c|c|c|c|c|c|}
\hline $0^{\prime}$ & 3.88577 & $25^{\circ}$ & $0^{\prime} 978.95388$ & $26^{\circ}$ & 9.02402 & $27^{\circ}$ & 9.09611 \\
\hline $\mathbf{I}^{\prime}$ & .88689 & & $\mathrm{l}^{\prime} \quad .95503$ & $1^{\prime}$ & .02521 & $1^{\prime}$ & .09733 \\
\hline $2^{\prime}$ & .88801 & & .95618 & $2^{\prime}$ & .02639 & $2^{\prime}$ & .09854 \\
\hline $3^{\prime}$ & .88913 & & .95734 & $3^{\prime}$ & .02758 & $3^{\prime}$ & .09976 \\
\hline $4^{\prime}$ & .89025 & & .95849 & 4 & .02877 & $4^{\prime}$ & .10098 \\
\hline $5^{\prime}$ & .89137 & & .95965 & $5^{\prime}$ & .02995 & $5^{\prime}$ & .10220 \\
\hline $6^{\prime}$ & .89249 & & .96080 & $6^{\prime}$ & .03114 & $6^{\prime}$ & .10342 \\
\hline $7^{\prime}$ & .89361 & & .96196 & $7^{\prime}$ & .03233 & $\tau^{\prime}$ & .10464 \\
\hline $8^{\prime}$ & .89473 & & .96312 & $8^{\prime}$ & .03352 & $8^{\prime}$ & .10586 \\
\hline $9^{\prime}$ & .89585 & & .96427 & $9^{\prime}$ & .03471 & $9^{\prime}$ & .10708 \\
\hline $10^{\prime}$ & .89698 & & .9654 .3 & $10^{\prime}$ & .03590 & $10^{\prime}$ & 10831 \\
\hline $11^{\prime}$ & .89810 & & .96659 & $11^{\prime}$ & .03709 & ' & .10953 \\
\hline $12^{\prime}$ & .89923 & & .96775 & $12^{\prime}$ & .03828 & $12^{\prime}$ & .11075 \\
\hline $13^{\prime}$ & .90035 & & .96891 & $13^{\prime}$ & .03948 & $13^{\prime}$ & .11198 \\
\hline $14^{\prime}$ & .90148 & & .97007 & $14^{\prime}$ & .04067 & $4^{\prime}$ & .11320 \\
\hline $15^{\prime}$ & .90260 & & .97123 & $15^{\prime}$ & .04186 & $15^{\prime}$ & .11442 \\
\hline $16^{\prime}$ & .90373 & & .97239 & $16^{\prime}$ & .04306 & $16^{\prime}$ & .11565 \\
\hline $17^{\prime}$ & .90486 & & .97355 & $17^{\prime}$ & .04425 & $17^{\prime}$ & 11688 \\
\hline $18^{\prime}$ & .90598 & & $.97+71$ & $18^{\prime}$ & .04545 & 18 & .11810 \\
\hline $19^{\prime}$ & .90711 & & .97587 & $19^{\prime}$ & .04664 & $19^{\prime}$ & .11933 \\
\hline $20^{\prime}$ & .90824 & & .97704 & $20^{\prime}$ & .04784 & $20^{\prime}$ & .12056 \\
\hline $21^{\prime}$ & .90937 & & .97820 & $21^{\prime}$ & .04903 & $21^{\prime}$ & .12178 \\
\hline $22^{\prime}$ & .91050 & & .97937 & $22^{\prime}$ & .05023 & $22^{\prime}$ & $.1230 \mid$ \\
\hline $23^{\prime}$ & .91163 & & .98053 & $23^{\prime}$ & .05143 & $23^{\prime}$ & .12424 \\
\hline $24^{\prime}$ & .91276 & & .98170 & $24^{\prime}$ & .05263 & $24^{\prime}$ & .12547 \\
\hline $25^{\prime}$ & .91390 & & .98286 & $25^{\prime}$ & .05382 & $25^{\prime}$ & .12670 \\
\hline $26^{\prime}$ & .91503 & & .98403 & $26^{\prime}$ & .05502 & $26^{\prime}$ & .12793 \\
\hline $27^{\prime}$ & .91616 & & .98520 & $27^{\prime}$ & .05622 & $27^{\prime}$ & 12916 \\
\hline $28^{\prime}$ & .91730 & & .98636 & $28^{\prime}$ & .05742 & $28^{\prime}$ & .13039 \\
\hline $29^{\prime}$ & .91843 & & .98753 & $29^{\prime}$ & .05862 & $29^{\prime}$ & 13162 \\
\hline $30^{\prime}$ & .91957 & & .98870 & $30^{\prime}$ & .05983 & $30^{\prime}$ & .13286 \\
\hline $3 I^{\prime}$ & .92070 & & .98987 & $31^{\prime}$ & .06103 & $31^{\prime}$ & 13409 \\
\hline $32^{\prime}$ & .92184 & & .99104 & $32^{\prime}$ & .06223 & $32^{\prime}$ & .13532 \\
\hline $33^{\prime}$ & .92297 & & .99221 & $33^{\prime}$ & .06343 & $33^{\prime}$ & .13656 \\
\hline $34^{\prime}$ & .92411 & & .09338 & $34^{\prime}$ & .06464 & $34^{\prime}$ & .13779 \\
\hline $35^{\prime}$ & .92525 & & .99455 & $35^{\prime}$ & .06584 & $35^{\prime}$ & .13903 \\
\hline $36^{\prime}$ & .92639 & & .99572 & $36^{\prime}$ & .06704 & $36^{\prime}$ & .14026 \\
\hline $37^{\prime}$ & .92753 & & .99690 & $37^{\prime}$ & .06825 & $37^{\prime}$ & $.1+150$ \\
\hline $38^{\prime}$ & .92867 & & .99807 & $38^{\prime}$ & .06946 & $38^{\prime}$ & .14273 \\
\hline $39^{\prime}$ & .92981 & & $39^{\prime} \quad .99924$ & $39^{\prime}$ & .07066 & $39^{\prime}$ & .14397 \\
\hline $40^{\prime}$ & .93095 & & $40^{\prime} 979.00042$ & $40^{\prime}$ & .07187 & $40)^{\prime}$ & .14521 \\
\hline $41^{\prime}$ & .93209 & & $41^{\prime} \quad .00159$ & $41^{\prime}$ & .07307 & $41^{\prime}$ & $.146 \pm 5$ \\
\hline $42^{\prime}$ & .93323 & & .00277 & $42^{\prime}$ & .07418 & $42^{\prime}$ & .14768 \\
\hline $43^{\prime}$ & .93437 & & .00394 & $43^{\prime}$ & .07549 & $43^{\prime}$ & .14892 \\
\hline $44^{\prime}$ & .93551 & & .00512 & $44^{\prime}$ & .07670 & $44^{\prime}$ & .15016 \\
\hline $45^{\prime}$ & .93666 & & .00630 & $45^{\prime}$ & .07791 & $45^{\prime}$ & .15140 \\
\hline $46^{\prime}$ & .93780 & & .00748 & $46^{\prime}$ & .07912 & $46^{\prime}$ & .15264 \\
\hline $47^{\prime}$ & .93895 & & .00865 & $47^{\prime}$ & .08033 & $47^{\prime}$ & .15388 \\
\hline $48^{\prime}$ & .94009 & & .00983 & $48^{\prime}$ & .08154 & $48^{\prime}$ & .15512 \\
\hline $49^{\prime}$ & 94124 & & 0101 & $49^{\prime}$ & .08275 & $49^{\prime}$ & .15637 \\
\hline $50^{\prime}$ & .94238 & & . () 1219 & $50^{\prime}$ & .08396 & $50)^{\prime}$ & .15761 \\
\hline $51^{\prime}$ & .94353 & & 01337 & $51^{\prime}$ & .08517 & $51^{\prime}$ & .15885 \\
\hline $52^{\prime}$ & .94468 & & .01455 & $52^{\prime}$ & .08639 & $52^{\prime}$ & 16009 \\
\hline $53^{\prime}$ & .94583 & & .01573 & $53^{\prime}$ & .08760 & $53^{\prime}$ & .16134 \\
\hline $54^{\prime}$ & 94698 & & .01692 & $54^{\prime}$ & .08881 & $54^{\prime}$ & 16258 \\
\hline $55^{\prime}$ & .94812 & & . & $55^{\prime}$ & .09003 & $55^{\prime}$ & 16383 \\
\hline $56^{\prime}$ & .94927 & & . () 1928 & $56^{\prime}$ & .09124 & $50^{\prime}$ & .16507 \\
\hline $57^{\prime}$ & .95042 & & .02047 & $57^{\prime}$ & .09246 & $57^{\prime}$ & 16632 \\
\hline $58^{\prime}$ & .95158 & & .02165 & $58^{\prime}$ & .09367 & $58^{\prime}$ & 16756 \\
\hline $59^{\prime}$ & .95273 & & .02283 & $59^{\prime}$ & .09489 & $59^{\prime}$ & .16881 \\
\hline
\end{tabular}


Table I - (continued)

\begin{tabular}{|c|c|c|c|c|c|c|c|}
\hline $0^{\prime}$ & 9.17006 & $29^{n}$ & 9.24578 & $30^{\circ}$ & $0^{\prime} 979.32318$ & $31^{\circ}$ & 9.40217 \\
\hline $1^{\prime}$ & .17131 & $I^{\prime}$ & .24706 & & $\mathrm{I}^{\prime} \quad .32449$ & $l^{\prime}$ & .40350 \\
\hline $2^{\prime}$ & .17255 & $2^{\prime}$ & .24833 & & .32579 & $2^{\prime}$ & .40483 \\
\hline $3^{\prime}$ & .17380 & $3^{\prime}$ & $.2\lfloor 961$ & & .32710 & $3^{\prime}$ & .40616 \\
\hline $4^{\prime}$ & .17505 & $4^{\prime}$ & .25089 & & .32840 & $4^{\prime}$ & 40749 \\
\hline $5^{\prime}$ & .17630 & $5^{\prime}$ & .25217 & & .32971 & $5^{\prime}$ & 40882 \\
\hline $6^{\prime}$ & .17755 & $6^{\prime}$ & .25345 & & .33101 & $6^{\prime}$ & .41015 \\
\hline $7^{\prime}$ & .17880 & $7^{\prime}$ & .25473 & & .33232 & $7^{\prime}$ & 41149 \\
\hline $8^{\prime}$ & .18005 & $8^{\prime}$ & $.256(6) 1$ & & .33362 & $8^{\prime}$ & .41282 \\
\hline $9^{\prime}$ & .18131 & $9^{\prime}$ & .25729 & & .33493 & $9^{\prime}$ & .41415 \\
\hline $10^{\prime}$ & .18256 & $10^{\prime}$ & .25857 & & .33624 & $10^{\prime}$ & .41548 \\
\hline $1 \mathrm{I}^{\prime}$ & .18381 & $\mathrm{II}^{\prime}$ & .25985 & & .33755 & $11^{\prime}$ & .41682 \\
\hline $12^{\prime}$ & .18506 & $12^{\prime}$ & .26113 & & .33886 & $12^{\prime}$ &.+1815 \\
\hline $13^{\prime}$ & .18632 & $13^{\prime}$ & .26241 & & .34017 & $13^{\prime}$ &.+1949 \\
\hline $14^{\prime}$ & .18757 & $14^{\prime}$ & .26369 & & .34147 & $14^{\prime}$ & .42082 \\
\hline $15^{\prime}$ & .18883 & $15^{\prime}$ & .26498 & & .34278 & $15^{\prime}$ & .42215 \\
\hline $16^{\prime}$ & .19008 & $16^{\prime}$ & .266626 & & .34409 & $166^{\prime}$ & $.+23+9$ \\
\hline $17^{\prime}$ & .19134 & $17^{\prime}$ & .26754 & & $.345+1$ & $17^{\prime}$ & .42483 \\
\hline $18^{\prime}$ & .19259 & $18^{\prime}$ & .26883 & & .34662 & $18^{\prime}$ &.+2616 \\
\hline $19^{\prime}$ & .19385 & $19^{\prime}$ & .27011 & & .34803 & $19^{\prime}$ &.+2750 \\
\hline 20$)^{\prime}$ & .19511 & $20^{\prime}$ & .27140 & & .34934 & $20^{\prime}$ & .42884 \\
\hline $2 \mathrm{l}^{\prime}$ & .19636 & $2 I^{\prime}$ & .27268 & & .35065 & $21^{\prime}$ & .43017 \\
\hline $22^{\prime}$ & .19762 & $22^{\prime}$ & .27397 & & .35197 & $22^{\prime}$ & .43151 \\
\hline $23^{\prime}$ & .19888 & $23^{\prime}$ & .27526 & & 35328 & $23^{\prime}$ & 43285 \\
\hline $24^{\prime}$ & .20014 & $24^{\prime}$ & .27654 & & .35459 & $24^{\prime}$ & .43419 \\
\hline $25^{\prime}$ & .20140 & $25^{\prime}$ & .27783 & & .35591 & $25^{\prime}$ & 43553 \\
\hline $26^{\prime}$ & 20266 & $26^{\prime}$ & .27912 & & 35722 & $26^{\prime}$ & .43687 \\
\hline $27^{\prime}$ & .20392 & $27^{\prime}$ & .28041 & & .35854 & $27^{\prime}$ & .43821 \\
\hline $28^{\prime}$ & .20 .518 & $28^{\prime}$ & .28170 & & .35985 & $28^{\prime}$ & .43955 \\
\hline $29^{\prime}$ & .20644 & $29^{\prime}$ & .28299 & & .36117 & $29^{\prime}$ & .44089 \\
\hline $30^{\prime}$ & .20770 & $30^{\prime}$ & 28428 & & 36248 & $30)^{\prime}$ & .44223 \\
\hline $31^{\prime}$ & .20897 & $31^{\prime}$ & .28557 & & .36380 & $31^{\prime}$ & .44357 \\
\hline $32^{\prime}$ & 21023 & $32^{\prime}$ & 28686 & & 36512 & $32^{\prime}$ & $4+491$ \\
\hline $33^{\prime}$ & .21149 & $33^{\prime}$ & .28815 & & .36644 & $33^{\prime}$ & .44626 \\
\hline $34^{\prime}$ & .21276 & $34^{\prime}$ & .28944 & & 36775 & $34^{\prime}$ & $\begin{array}{r}4760 \\
\end{array}$ \\
\hline $355^{\prime}$ & .21402 & $35^{\prime}$ & .29073 & & .36907 & $35^{\prime}$ & .44894 \\
\hline $36^{\prime}$ & .21528 & $36^{\prime}$ & 29203 & & 37039 & $36^{\prime}$ & 45029 \\
\hline $37^{\prime}$ & .21655 & $37^{\prime}$ & .29332 & 37 & .37171 & $37^{\prime}$ & .45163 \\
\hline $38^{\prime}$ & .21782 & $38^{\prime}$ & 29461 & 38 & 37303 & $38^{\prime}$ &.+5297 \\
\hline $39^{\prime}$ & 21908 & $39^{\prime}$ & .29591 & & .37435 & $39^{\prime}$ & .45432 \\
\hline $40^{\prime}$ & .22035 & $40^{\prime}$ & .29720 & 40 & .37567 & 4()$^{\prime}$ & 45566 \\
\hline $4 l^{\prime}$ & .22162 & $41^{\prime}$ & .29850 & 41 & 37699 & $41^{\prime}$ & .45701 \\
\hline $42^{\prime}$ & 22288 & $42^{\prime}$ & .29979 & 42 & 37831 & $42^{\prime}$ & .45836 \\
\hline $43^{\prime}$ & .22415 & $43^{\prime}$ & .30109 & 43 & 37964 & $43^{\prime}$ &.+5970 \\
\hline $44^{\prime}$ & .22542 & $44^{\prime}$ & 30238 & & 38096 & $44^{\prime}$ & .46105 \\
\hline $45^{\prime}$ & .226699 & $45^{\prime}$ & .30368 & 45 & 38228 & $45^{\prime}$ & .46240 \\
\hline $46^{\prime}$ & .22796 & $46^{\prime}$ & .30498 & & 38360 & $46^{\prime}$ & .46374 \\
\hline $47^{\prime}$ & .22923 & $47^{\prime}$ & .30627 & 47 & .38493 & $47^{\prime}$ & .46509 \\
\hline $48^{\prime}$ & .23050 & $48^{\prime}$ & .30757 & & 38625 & $48^{\prime}$ & .46644 \\
\hline $49^{\prime}$ & .23177 & $49^{\prime}$ & .30887 & 49 & .38758 & $49^{\prime}$ & .46779 \\
\hline 50$)^{\prime}$ & .23304 & $50^{\prime}$ & .31017 & & .38890 & $50^{\prime}$ & .46914 \\
\hline $5 I^{\prime}$ & .23431 & $5 l^{\prime}$ & .31147 & & .39023 & $51^{\prime}$ & .47049 \\
\hline $52^{\prime}$ & 23559 & $52^{\prime}$ & .31277 & & 39155 & $52^{\prime}$ & .47184 \\
\hline $53^{\prime}$ & .23686 & $53^{\prime}$ & .31407 & & 39288 & $53^{\prime}$ & .47319 \\
\hline $54^{\prime}$ & .23813 & $54^{\prime}$ & 31537 & & .39420 & $54^{\prime}$ & 47454 \\
\hline $55^{\prime}$ & $.239+0$ & $55^{\prime}$ & .31667 & & .39553 & $55^{\prime}$ & .47589 \\
\hline $56^{\prime}$ & 24068 & $56^{\prime}$ & 31797 & & .39686 & $56^{\prime}$ & 47724 \\
\hline $57^{\prime}$ & .24195 & $57^{\prime}$ & .31927 & & 39819 & $57^{\prime}$ &.+7859 \\
\hline $58^{\prime}$ & .24323 & $58^{\prime}$ & 32058 & & 39951 & $58^{\prime}$ & .47994 \\
\hline $59^{\prime}$ & .24450 & $59^{\prime}$ & .32188 & & .40084 & $59^{\prime}$ & 48130 \\
\hline
\end{tabular}


Table I - (continued)

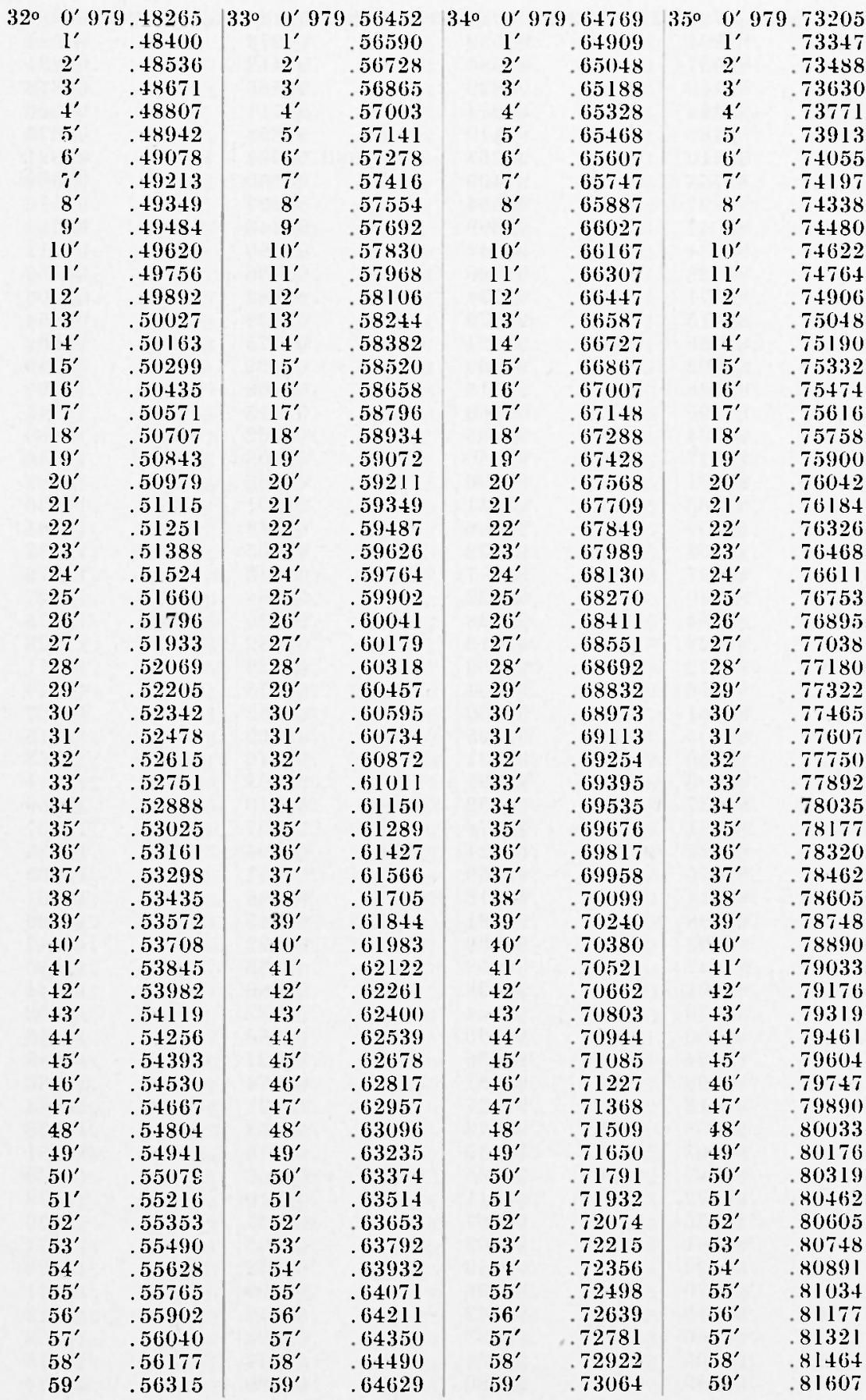


Table I - (continued)

\begin{tabular}{|c|c|c|c|c|c|c|c|}
\hline $36^{\circ}$ & .81750 & $37^{\circ}$ & $0^{\prime} 979.90394$ & $38^{\circ}$ & $0^{\prime} 979.99126$ & $39^{\circ}$ & 30.07936 \\
\hline$I^{\prime}$ & .81894 & & $l^{\prime} \quad .90539$ & & $\mathrm{I}^{\prime} \quad .99273$ & $\mathrm{l}^{\prime}$ & .08084 \\
\hline $2^{\prime}$ & .82037 & & .90684 & & $.99+19$ & $2^{\prime}$ & .08231 \\
\hline $3^{\prime}$ & .82180 & & .90829 & & 99565 & $3^{\prime}$ & .08378 \\
\hline $4^{\prime}$ & .82324 & & .90974 & & 99711 & $4^{\prime}$ & .08526 \\
\hline $5^{\prime}$ & .82467 & & .91119 & & $5^{\prime} \quad .99858$ & $5^{\prime}$ & .08673 \\
\hline $6^{\prime}$ & .82610 & & .91264 & & $6^{\prime} 980.00004$ & $6^{\prime}$ & .08821 \\
\hline $7^{\prime}$ & .82754 & & .91409 & & $7^{\prime} \quad .00150$ & $7^{\prime}$ & .08969 \\
\hline $8^{\prime}$ & .82897 & & .91554 & & .00297 & $8^{\prime}$ & .09116 \\
\hline $9^{\prime}$ & .83041 & & .91699 & & .00443 & $9^{\prime}$ & .09264 \\
\hline $10^{\prime}$ & .83184 & $10^{\prime}$ & .91844 & & .00590 & $10^{\prime}$ & .09411 \\
\hline $11^{\prime}$ & .83328 & $11^{\prime}$ & .91989 & & .00736 & $11^{\prime}$ & .09559 \\
\hline $12^{\prime}$ & .83471 & $12^{\prime}$ & .92134 & & .00882 & $12^{\prime}$ & .09706 \\
\hline $13^{\prime}$ & .83615 & $13^{\prime}$. & .92279 & & .01029 & $13^{\prime}$ & .09854 \\
\hline $14^{\prime}$ & .83759 & $14^{\prime}$ & .92424 & & .01175 & $14^{\prime}$ & .10002 \\
\hline $15^{\prime}$ & .83902 & $15^{\prime}$ & .92569 & & .01322 & $15^{\prime}$ & .10149 \\
\hline $16^{\prime}$ & .84046 & $16^{\prime}$ & .92715 & & .01468 & $16^{\prime}$ & .10297 \\
\hline $17^{\prime}$ & .84190 & $17^{\prime}$ & .92860 & & .01615 & $17^{\prime}$ & .10445 \\
\hline $18^{\prime}$ & .84334 & $18^{\prime}$ & .93005 & & .01762 & $18^{\prime}$ & .10593 \\
\hline $19^{\prime}$ & .84477 & $19^{\prime}$ & .93150 & & .01908 & $19^{\prime}$ & .10740 \\
\hline $20^{\prime}$ & .84621 & $20^{\prime}$ & .93296 & & .02055 & $20^{\prime}$ & .10888 \\
\hline $2 \mathrm{I}^{\prime}$ & .84765 & $21^{\prime}$ & .93441 & & .02201 & $21^{\prime}$ & .11036 \\
\hline $22^{\prime}$ & .84909 & $22^{\prime}$ & .93586 & & .02348 & $22^{\prime}$ & .11184 \\
\hline $23^{\prime}$ & .85053 & $23^{\prime}$ & .93732 & & .02495 & $23^{\prime}$ & .11332 \\
\hline $24^{\prime}$ & .85197 & $24^{\prime}$ & .93877 & & .02642 & $24^{\prime}$ & $.11+79$ \\
\hline $25^{\prime}$ & .85340 & $25^{\prime}$ & .94022 & & .02788 & $25^{\prime}$ & .11627 \\
\hline $86^{\prime}$ & .85484 & $26^{\prime}$ & .94168 & & .02935 & $26^{\prime}$ & .11775 \\
\hline $27^{\prime}$ & .85628 & $27^{\prime}$ & .94313 & & .03082 & $27^{\prime}$ & .11923 \\
\hline $28^{\prime}$ & .85772 & $28^{\prime}$ & .94459 & & .03229 & $28^{\prime}$ & .12071 \\
\hline $29^{\prime}$ & .85916 & $29^{\prime}$ & .94604 & & .03375 & $29^{\prime}$ & .12219 \\
\hline $30^{\prime}$ & .86061 & $30^{\prime}$ & .94750 & & .03522 & $30^{\prime}$ & .12367 \\
\hline $3 I^{\prime}$ & .86205 & $3 I^{\prime}$ & .94895 & & .03669 & $31^{\prime}$ & .12515 \\
\hline $32^{\prime}$ & $863+9$ & $32^{\prime}$ & .95041 & & .03816 & $32^{\prime}$ & .12663 \\
\hline $33^{\prime}$ & $.86+93$ & $33^{\prime}$ & .95187 & & .03963 & $33^{\prime}$ & .12811 \\
\hline $34^{\prime}$ & .86637 & $34^{\prime}$ & .95332 & & .04110 & $34^{\prime}$ & .12959 \\
\hline $35^{\prime}$ & .86781 & $35^{\prime}$ & .95478 & & .04257 & $35^{\prime}$ & .13107 \\
\hline $36^{\prime}$ & .86925 & $36^{\prime}$ & .95624 & & .04404 & $36^{\prime}$ & .13255 \\
\hline $37^{\prime}$ & .87070 & $37^{\prime}$ & .95769 & & .04551 & $37^{\prime}$ & .13403 \\
\hline $38^{\prime}$ & .87214 & $38^{\prime}$ & .95915 & & .04698 & $38^{\prime}$ & .13551 \\
\hline $39^{\prime}$ & .87358 & $39^{\prime}$ & .96061 & & .04845 & $39^{\prime}$ & 13699 \\
\hline $40^{\prime}$ & .87503 & $40^{\prime}$ & .96206 & & .04992 & $40^{\prime}$ & .13847 \\
\hline$+I^{\prime}$ & .87647 & $41^{\prime}$ & .96352 & & .05139 & $41^{\circ}$ & .13995 \\
\hline $42^{\prime}$ & .87791 & $42^{\prime}$ & .96498 & & .05286 & $42^{\prime}$ & .14144 \\
\hline $43^{\prime}$ & .87936 & $43^{\prime}$ & .96644 & & .05433 & $43^{\prime}$ & .14292 \\
\hline $44^{\prime}$ & .88080 & $44^{\prime}$ & .96790 & & .05580 & $44^{\prime}$ & .14440 \\
\hline $45^{\prime}$ & .88224 & $45^{\prime}$ & .96936 & & .05727 & $45^{\prime}$ & .14588 \\
\hline $46^{\prime}$ & .88369 & $46^{\prime}$ & .97081 & & .05874 & $46^{\prime}$ & .14736 \\
\hline $47^{\prime}$ & .88513 & $47^{\prime}$ & .97227 & & .06021 & $47^{\prime}$ & .14884 \\
\hline $48^{\prime}$ & .88658 & $48^{\prime}$ & .97373 & & .06168 & $48^{\prime}$ & .15033 \\
\hline $49^{\prime}$ & .88803 & $49^{\prime}$ & .97519 & & .06316 & $49^{\prime}$ & .15181 \\
\hline $50^{\prime}$ & .88947 & $50^{\prime}$ & .97665 & & .06463 & $50^{\prime}$ & .15329 \\
\hline $5 I^{\prime}$ & .89092 & $5 \mathrm{l}^{\prime}$ & .97811 & & .06610 & $5 l^{\prime}$ & .15478 \\
\hline $52^{\prime}$ & .89236 & $52^{\prime}$ & .97957 & & .06757 & $52^{\prime}$ & .15626 \\
\hline $53^{\prime}$ & .89381 & $53^{\prime}$ & .98103 & & .06905 & $53^{\prime}$ & .15774 \\
\hline $54^{\prime}$ & .89526 & $54^{\prime}$ & .98249 & & .07052 & $54^{\prime}$ & .15923 \\
\hline $55^{\prime}$ & .89670 & $55^{\prime}$ & .98396 & & .07199 & $55^{\prime}$ & .16071 \\
\hline $56^{\prime}$ & .89815 & $56^{\prime}$ & .98542 & & .07347 & $56^{\prime}$ & .16219 \\
\hline $57^{\prime}$ & .89960 & $57^{\prime}$ & .98688 & & .07494 & $57^{\prime}$ & .16368 \\
\hline $58^{\prime}$ & .90105 & $58^{\prime}$ & .98834 & & .07641 & $58^{\prime}$ & .16516 \\
\hline $59^{\prime}$ & .90249 & $59^{\prime}$ & .98980 & & .07789 & $59^{\prime}$ & 16664 \\
\hline
\end{tabular}


Table I - (coutinued)

\begin{tabular}{|c|c|c|c|c|c|c|c|}
\hline $40^{\circ}$ & .16813 & $41^{\circ}$ & $0^{\prime} 980.25746$ & $42^{\circ}$ & $J^{\prime} 980.34724$ & $+3^{\circ}$ & 0.43737 \\
\hline $\mathrm{l}^{\prime}$ & .16961 & & $1^{\prime} \quad .25895$ & $l^{\prime}$ & $1^{\prime} \quad .34874$ & $\mathrm{l}^{\prime}$ & .43887 \\
\hline $2^{\prime}$ & .17110 & & .26044 & $2^{\prime}$ & .35024 & $2^{\prime}$ & 44038 \\
\hline $3^{\prime}$ & .17258 & & .26194 & $3^{\prime}$ & .35174 & 3 & .44188 \\
\hline $4^{\prime}$ & .17407 & & 26343 & $4^{\prime}$ & .35324 & $t^{\prime}$ & 44339 \\
\hline $5^{\prime}$ & .17555 & & .26492 & $5^{\prime}$ & .35474 & $5^{\prime}$ & 44489 \\
\hline $6^{\prime}$ & .17704 & & .26642 & $6^{\prime}$ & .35624 & $6^{\prime}$ & .44640 \\
\hline $7^{\prime}$ & .17852 & & .26791 & $7^{\prime}$ & .35774 & $7^{\prime}$ & .44790 \\
\hline $8^{\prime}$ & $.1800 \mathrm{I}$ & & .26941 & $8^{\prime}$ & .35924 & $8^{\prime}$ & .44940 \\
\hline $9^{\prime}$ & .18150 & & .27090 & $9^{\prime}$ & .36074 & $9^{\prime}$ & .45091 \\
\hline $10^{\prime}$ & .18298 & & .27239 & $10^{\prime}$ & .36224 & $10^{\prime}$ & .45241 \\
\hline $11^{\prime}$ & .18447 & & .27389 & $11^{\prime}$ & .36374 & $11^{\prime}$ & .45392 \\
\hline $12^{\prime}$ & .18595 & & .27538 & $12^{\prime}$ & .36524 & $12^{\prime}$ & .45542 \\
\hline $13^{\prime}$ & .18744 & & .27688 & $13^{\prime}$ & .36674 & $13^{\prime}$ & .45693 \\
\hline $14^{\prime}$ & .18893 & & .27837 & $14^{\prime}$ & .36824 & $14^{\prime}$ & .45844 \\
\hline $15^{\prime}$ & .19041 & & .27987 & $15^{\prime}$ & .36975 & $15^{\prime}$ & .45994 \\
\hline $16^{\prime}$ & .19190 & & .28136 & $16^{\prime}$ & .37125 & $16^{\prime}$ & $.461+5$ \\
\hline $17^{\prime}$ & .19339 & & .28286 & $17^{\prime}$ & 37275 & $17^{\prime}$ & .46295 \\
\hline $18^{\prime}$ & .19487 & & .28435 & $18^{\prime}$ & .37425 & $18^{\prime}$ & .46446 \\
\hline $19^{\prime}$ & .19636 & & .28585 & $19^{\prime}$ & .37575 & $19^{\prime}$ & .46596 \\
\hline $20^{\prime}$ & .19785 & & .28734 & $20^{\prime}$ & .37725 & $20^{\prime}$ & .46747 \\
\hline 2$]^{\prime}$ & .19934 & & .28884 & $21^{\prime}$ & .37875 & $21^{\prime}$ & 46897 \\
\hline $22^{\prime}$ & .20082 & & .29033 & $22^{\prime}$ & .38025 & $22^{\prime}$ & .47048 \\
\hline $23^{\prime}$ & .20231 & & .29183 & $23^{\prime}$ & .38176 & $23^{\prime}$ & .47198 \\
\hline $24^{\prime}$ & 20380 & & .29332 & $24^{\prime}$ & .38326 & $24^{\prime}$ & .47349 \\
\hline $25^{\prime}$ & 20529 & & .29482 & $25^{\prime}$ & .38476 & $25^{\prime}$ & .47500 \\
\hline $26^{\prime}$ & .20678 & & .29632 & $26^{\prime}$ & .38626 & $26^{\prime}$ &.+7650 \\
\hline $27^{\prime}$ & 20826 & & .29781 & $27^{\prime}$ & .38776 & $27^{\prime}$ & .47801 \\
\hline $28^{\prime}$ & .20975 & & .29931 & $28^{\prime}$ & .38926 & $28^{\prime}$ & .47951 \\
\hline $29^{\prime}$ & .21124 & & .30080 & $29^{\prime}$ & 39077 & $29^{\prime}$ & .48102 \\
\hline $30^{\prime}$ & .21273 & & .30230 & $30^{\prime}$ & 39227 & $30^{\prime}$ & 48253 \\
\hline $31^{\prime}$ & .21422 & & .30380 & $31^{\prime}$ & .39377 & $31^{\prime}$ & .48403 \\
\hline $32^{\prime}$ & .2157 & & .30529 & $32^{\prime}$ & .39527 & $32^{\prime}$ & .48554 \\
\hline $33^{\prime}$ & .21720 & & .30679 & $33^{\prime}$ & .39678 & $33^{\prime}$ & .48704 \\
\hline $24^{\prime}$ & .21869 & & .30829 & $34^{\prime}$ & .39828 & $34^{\prime}$ & .48855 \\
\hline $35^{\prime}$ & .22018 & & .30978 & $35^{\prime}$ & .39978 & $35^{\prime}$ & .49006 \\
\hline $36^{\prime}$ & .22167 & & .31128 & $36^{\prime}$ & .40128 & $36^{\prime}$ & .49156 \\
\hline $37^{\prime}$ & .22316 & & .31278 & $37^{\prime}$ & .40279 & $37^{\prime}$ & .49307 \\
\hline $38^{\prime}$ & .22465 & & .31428 & $38^{\prime}$ & .40429 & $38^{\prime}$ & .49458 \\
\hline $39^{\prime}$ & .22614 & & .31577 & $39^{\prime}$ & .40579 & $39^{\prime}$ & .49608 \\
\hline $40^{\prime}$ & .22763 & & 31727 & $40^{\prime}$ & .40729 & $40^{\prime}$ & .49759 \\
\hline $41^{\prime}$ & .22912 & & .31877 & $4 l^{\prime}$ & .40880 & $41^{\prime}$ & .49910 \\
\hline $42^{\prime}$ & .23061 & & .32027 & $42^{\prime}$ & .41030 & $42^{\prime}$ & .50060 \\
\hline $43^{\prime}$ & .23210 & & .32176 & $43^{\prime}$ & .41180 & $43^{\prime}$ & .50211 \\
\hline $44^{\prime}$ & .23359 & & .32326 & $44^{\prime}$ & .41331 & $44^{\prime}$ & .50362 \\
\hline $45^{\prime}$ & .23508 & & .32476 & $45^{\prime}$ & .41481 & $45^{\prime}$ & .50512 \\
\hline $46^{\prime}$ & .23657 & & 32626 & $46^{\prime}$ & 41631 & $46^{\prime}$ & .50663 \\
\hline $47^{\prime}$ & .23806 & & .32776 & $47^{\prime}$ & .41782 & $47^{\prime}$ & .50814 \\
\hline $48^{\prime}$ & .23955 & & .32925 & $48^{\prime}$ & .419 & $48^{\prime}$ & .50964 \\
\hline $49^{\prime}$ & .24104 & & .33075 & $49^{\prime}$ & .42082 & $49^{\prime}$ & .51115 \\
\hline $50^{\prime}$ & .24254 & & .33225 & $50^{\prime}$ & .42233 & $50^{\prime}$ & .51266 \\
\hline $5 l^{\prime}$ & .24403 & & .33375 & $51^{\prime}$ & .42383 & $5 l^{\prime}$ & .51416 \\
\hline $52^{\prime}$ & .24552 & & .33525 & $52^{\prime}$ & .42534 & $52^{\prime}$ & .51567 \\
\hline $53^{\prime}$ & .24701 & & .33675 & $53^{\prime}$ & .42684 & $53^{\prime}$ & .51718 \\
\hline $54^{\prime}$ & .24850 & & .33825 & $54^{\prime}$ & .42834 & $54^{\prime}$ & .51869 \\
\hline $55^{\prime}$ & .25000 & & .33974 & $55^{\prime}$ & .42985 & $55^{\prime}$ & .52019 \\
\hline $56^{\prime}$ & .25149 & & .34124 & $56^{\prime}$ & .43135 & $56^{\prime}$ & .52170 \\
\hline $57^{\prime}$ & .25298 & & .34274 & $57^{\prime}$ & .43286 & $57^{\prime}$ & .52321 \\
\hline $58^{\prime}$ & .25447 & & .34424 & $58^{\prime}$ & .43436 & $58^{\prime}$ & .52471 \\
\hline $59^{\prime}$ & .25597 & & .34574 & $59^{\prime}$ & .43586 & $59^{\prime}$ & .52622 \\
\hline
\end{tabular}


Table I - (continued)

\begin{tabular}{|c|c|c|c|c|c|c|c|}
\hline $0^{\prime}$ & .52773 & $45^{\circ}$ & $0^{\prime} 980.61821$ & $46^{\circ}$ & $0^{\prime} 980.7087 \mathrm{I}$ & $47^{\circ}$ & )$^{\prime} 980.79911$ \\
\hline$I^{\prime}$ & .52924 & & $I^{\prime} \quad .61972$ & & $\mathrm{I}^{\prime} \quad .71022$ & $\mathbf{I}^{\prime}$ & .80062 \\
\hline $2^{\prime}$ & .53074 & & .62123 & & .71173 & $\mathbf{2}^{\prime}$ & .80213 \\
\hline $3^{\prime}$ & .53225 & & .62274 & & .71324 & $3^{\prime}$ & .80363 \\
\hline $4^{\prime}$ & .53376 & & .62425 & & $.71+74$ & $4^{\prime}$ & .80514 \\
\hline $5^{\prime}$ & .53527 & & .62576 & & .71625 & $5^{\prime}$ & .80664 \\
\hline $6^{\prime}$ & .53677 & & .62726 & & .71776 & $6^{\prime}$ & .80815 \\
\hline $7^{\prime}$ & .53828 & & .62877 & & .71927 & $7^{\prime}$ & .80965 \\
\hline $8^{\prime}$ & .53979 & & .63028 & & .72077 & $8^{\prime}$ & .81116 \\
\hline $9^{\prime}$ & .54130 & & .63179 & & .72228 & $9^{\prime}$ & .81266 \\
\hline $10^{\prime}$ & .54280 & & 63330 & $10^{\prime}$ & .72379 & $10^{\prime}$ & .81416 \\
\hline $\mathrm{II}^{\prime}$ & .54431 & & 63481 & $11^{\prime}$ & .72530 & $11^{\prime}$ & .81567 \\
\hline $11^{\prime}$ & .54582 & & .63632 & $12^{\prime}$ & .72680 & $12^{\prime}$ & .81717 \\
\hline $13^{\prime}$ & .54733 & & .63782 & $13^{\prime}$ & .72831 & $13^{\prime}$ & .81868 \\
\hline $14^{\prime}$ & .54883 & & .63933 & $14^{\prime}$ & .72982 & $14^{\prime}$ & .82018 \\
\hline $15^{\prime}$ & .55034 & & .64084 & $15^{\prime}$ & .73133 & $15^{\prime}$ & .82169 \\
\hline $16^{\prime}$ & .55185 & & .64235 & $16^{\prime}$ & .73283 & $16^{\prime}$ & .82319 \\
\hline $17^{\prime}$ & .55336 & & .64386 & $17^{\prime}$ & .73434 & $17^{\prime}$ & .82470 \\
\hline $18^{\prime}$ & .55487 & & .64537 & $18^{\prime}$ & .73589 & $18^{\prime}$ & .82620 \\
\hline $19^{\prime}$ & .55637 & & .64688 & $19^{\prime}$ & .73736 & $19^{\prime}$ & .82770 \\
\hline $20^{\prime}$ & .55788 & & .64838 & $20^{\prime}$ & .73886 & $20^{\prime}$ & .82921 \\
\hline $21^{\prime}$ & .55939 & & .64989 & $21^{\prime}$ & .74037 & $21^{\prime}$ & .83071 \\
\hline $22^{\prime}$ & .56090 & & .65140 & $22^{\prime}$ & $.7+188$ & $22^{\prime}$ & .83222 \\
\hline $23^{\prime}$ & .56241 & & 65291 & $23^{\prime}$ & .74338 & $23^{\prime}$ & .83372 \\
\hline $24^{\prime}$ & .56391 & & $65+42$ & $24^{\prime}$ & .74489 & $24^{\prime}$ & 83522 \\
\hline $25^{\prime}$ & .56542 & & .65593 & $25^{\prime}$ & $.746+0$ & $25^{\prime}$ & 83673 \\
\hline $26^{\prime}$ & .56693 & & $657+3$ & $26^{\prime}$ & 74790 & $26^{\prime}$ & 83823 \\
\hline $27^{\prime}$ & .56844 & & 65894 & $27^{\prime}$ & $.749+1$ & $27^{\prime}$ & .83973 \\
\hline $28^{\prime}$ & .56995 & & 66045 & $28^{\prime}$ & .75092 & $28^{\prime}$ & 84124 \\
\hline $29^{\prime}$ & $.57 \mid 45$ & & 66196 & $29^{\prime}$ & .75243 & $29^{\prime}$ & 84274 \\
\hline $30^{\prime}$ & .57296 & & .66347 & $30)^{\prime}$ & .75393 & $30^{\prime}$ & 84425 \\
\hline $31^{\prime}$ & .57447 & & $66+98$ & $3 l^{\prime}$ & .75544 & $31^{\prime}$ & 84575 \\
\hline $32^{\prime}$ & .57598 & & .66648 & $32^{\prime}$ & .75695 & $32^{\prime}$ & 84725 \\
\hline $33^{\prime}$ & .57749 & & .66799 & $33^{\prime}$ & .75845 & $33^{\prime}$ & 84876 \\
\hline $34^{\prime}$ & .57900 & & 66950 & $34^{\prime}$ & .75996 & $34^{\prime}$ & 85026 \\
\hline $3 \tilde{5}^{\prime}$ & .58050 & & .67101 & $35^{\prime}$ & .76147 & $35^{\prime}$ & .85176 \\
\hline $36^{\prime}$ & .58201 & & .67252 & $36^{\prime}$ & .76297 & $36^{\prime}$ & 85326 \\
\hline $37^{\prime}$ & .58352 & & .67403 & $37^{\prime}$ & .76 & $37^{\prime}$ & .85477 \\
\hline $38^{\prime}$ & .58503 & & .67553 & $38^{\prime}$ & .76599 & $38^{\prime}$ & 85627 \\
\hline $39^{\prime}$ & .58654 & & .67704 & $39^{\prime}$ & .76749 & $39^{\prime}$ & .85777 \\
\hline $40^{\prime}$ & .58804 & & .67855 & $40^{\prime}$ & .76900 & $40^{\prime}$ & .85928 \\
\hline $4 I^{\prime}$ & .58955 & & .68006 & $41^{\prime}$ & .77050 & $41^{\prime}$ & .86078 \\
\hline$+2^{\prime}$ & .59106 & & 68157 & $42^{\prime}$ & .77201 & $42^{\prime}$ & 86228 \\
\hline$+3^{\prime}$ & .59257 & & .68308 & $43^{\prime}$ & .77352 & $43^{\prime}$ & .86378 \\
\hline $44^{\prime}$ & .59408 & & 68458 & $44^{\prime}$ & .77502 & $44^{\prime}$ & .86508 \\
\hline $45^{\prime}$ & .59559 & & .68609 & $45^{\prime}$ & .77653 & $45^{\prime}$ & .86679 \\
\hline $46^{\prime}$ & .59709 & & 68760 & $46^{\prime}$ & .77804 & $46^{\prime}$ & 86829 \\
\hline $47^{\prime}$ & .59860 & & .68911 & $47^{\prime}$ & .77954 & $47^{\prime}$ & .86979 \\
\hline $48^{\prime}$ & .60011 & & .69062 & $48^{\prime}$ & .78105 & $48^{\prime}$ & .87129 \\
\hline $49^{\prime}$ & .60162 & & .69212 & $49^{\prime}$ & .78255 & $49^{\prime}$ & .87280 \\
\hline $50^{\prime}$ & 60313 & & .69363 & $50^{\prime}$ & 78406 & $50^{\prime}$ & .87430 \\
\hline $51^{\prime}$ & .60464 & & .69514 & $5 l^{\prime}$ & .78556 & $51^{\prime}$ & 87580 \\
\hline $52^{\prime}$ & 60615 & & .696655 & $52^{\prime}$ & .78707 & $52^{\prime}$ & .87730 \\
\hline $53^{\prime}$ & .60765 & & .69816 & $53^{\prime}$ & .78858 & $53^{\prime}$ & 87880 \\
\hline $54^{\prime}$ & .60916 & & 69966 & $54^{\prime}$ & .79008 & $54^{\prime}$ & .88030 \\
\hline $55^{\prime}$ & .61067 & & .70117 & $55^{\prime}$ & .79159 & $55^{\prime}$ & .88180 \\
\hline $56^{\prime}$ & .61218 & & .70268 & $56^{\prime}$ & .79309 & $56^{\prime}$ & .88331 \\
\hline $57^{\prime}$ & .61369 & & $.70+19$ & $57^{\prime}$ & .79460 & $57^{\prime}$ & $88+81$ \\
\hline $58^{\prime}$ & .61520 & & .70570 & $58^{\prime}$ & .79610 & $58^{\prime}$ & 88631 \\
\hline $59^{\prime}$ & .61670 & & .70720 & $59^{\prime}$ & .79761 & $59^{\prime}$ & .88781 \\
\hline
\end{tabular}


Table I - (continued)

\begin{tabular}{|c|c|c|c|c|c|c|c|}
\hline $4 \varepsilon^{\circ} \quad 0^{\prime}$ & 0.88931 & $49^{\circ}$ & ()'980.97919 & $50^{\circ}$ & $0^{\prime} 981.06864$ & $51^{\circ}$ & 1.15756 \\
\hline $\mathrm{I}^{\prime}$ & .89081 & & $l^{\prime} .98068$ & & ]$^{\prime} \quad .07013$ & $I^{\prime}$ & .15904 \\
\hline $2^{\prime}$ & .89231 & & .98218 & & .07162 & $2^{\prime}$ & .16051 \\
\hline $3^{\prime}$ & .89381 & & .98367 & & .07310 & 3 & .16199 \\
\hline $4^{\prime}$ & .89531 & & .98517 & & .07459 & $4^{\prime}$ & .16347 \\
\hline $5^{\prime}$ & .89681 & & .98666 & & .07608 & $5^{\prime}$ & .16494 \\
\hline $6^{\prime}$ & .89831 & & .98816 & & .07756 & $6^{\prime}$ & .16642 \\
\hline $7^{\prime}$ & .89981 & & .98965 & & .07905 & $\bar{t}^{\prime}$ & .16790 \\
\hline $8^{\prime}$ & .90132 & & .99114 & & .08053 & $8^{\prime}$ & .16937 \\
\hline $9^{\prime}$ & .90282 & & .99264 & & .08202 & $9^{\prime}$ & $.17(0355$ \\
\hline $10^{\prime}$ & .90432 & & $.99+13$ & $100^{\prime}$ & .08350 & $100^{\prime}$ & .17232 \\
\hline $11^{\prime}$ & .90582 & & .99562 & $11^{\prime}$ & .03499 & $11^{\prime}$ & .17380 \\
\hline $12^{\prime}$ & .90732 & & .99712 & $12^{\prime}$ & .08647 & $12^{\prime}$ & .17527 \\
\hline $13^{\prime}$ & .90882 & & .99861 & $13^{\prime}$ & .08796 & $13^{\prime}$ & .17675 \\
\hline $14^{\prime}$ & .91031 & & $14^{\prime} 981.00010$ & $14^{\prime}$ & $.089+4$ & $14^{\prime}$ & .17822 \\
\hline $15^{\prime}$ & .91181 & & $15^{\prime} \quad .00160$ & $15^{\prime}$ & .09093 & $15^{\prime}$ & .17969 \\
\hline $16^{\prime}$ & .91331 & & .00309 & $16^{\prime}$ & .092+1 & $16^{\prime}$ & .18117 \\
\hline $17^{\prime}$ & $.91+81$ & & .00458 & $17^{\prime}$ & .09390 & $17^{\prime}$ & .18264 \\
\hline $18^{\prime}$ & .91631 & & .00608 & $18^{\prime}$ & .09538 & $18^{\prime}$ & .18412 \\
\hline $19^{\prime}$ & .91781 & & .00757 & $19^{\prime}$ & .09686 & $19^{\prime}$ & .18559 \\
\hline $20^{\prime}$ & .91931 & & .00906 & $20)^{\prime}$ & .09835 & $20)^{\prime}$ & .18706 \\
\hline $21^{\prime}$ & .92081 & & .01055 & $21^{\prime}$ & .09983 & $21^{\prime}$ & .18854 \\
\hline $22^{\prime}$ & .92231 & & .01204 & $22^{\prime}$ & .10131 & $22^{\prime}$ & .19001 \\
\hline $23^{\prime}$ & .92381 & & .01354 & $23^{\prime}$ & .10280 & $23^{\prime}$ & .19148 \\
\hline $24^{\prime}$ & .92531 & & .01503 & $24^{\prime}$ & .10428 & $24^{\prime}$ & .19295 \\
\hline $25^{\prime}$ & .92681 & & .01652 & $25^{\prime}$ & .10576 & $25^{\prime}$ & $.19+43$ \\
\hline $26^{\prime}$ & .92830 & & .01801 & $26^{\prime}$ & .10725 & $26^{\prime}$ & .19590 \\
\hline $27^{\prime}$ & .92980 & & .01950 & $27^{\prime}$ & .10873 & $27^{\prime}$ & .19737 \\
\hline $28^{\prime}$ & .93130 & & .02099 & $38^{\prime}$ & .11021 & 28 & .19884 \\
\hline $29^{\prime}$ & .93280 & & .02249 & $29^{\prime}$ & .11169 & $29^{\prime}$ & .20031 \\
\hline $30^{\prime}$ & .93430 & & .02398 & $30)^{\prime}$ & .11318 & $300^{\prime}$ & .20179 \\
\hline $31^{\prime}$ & .93579 & & .02547 & $31^{\prime}$ & .11466 & $31^{\prime}$ & .20326 \\
\hline $32^{\prime}$ & .93729 & & .02696 & $32^{\prime}$ & .11614 & $32^{\prime}$ & .20473 \\
\hline $33^{\prime}$ & .93879 & & $.028+5$ & $33^{\prime}$ & .11762 & $33^{\prime}$ & .20620 \\
\hline $34^{\prime}$ & .04029 & & .02994 & $34^{\prime}$ & .11910 & $34^{\prime}$ & .20767 \\
\hline $35^{\prime}$ & $.9+179$ & & $.031+3$ & $35^{\prime}$ & . 12058 & $35^{\prime}$ & .20914 \\
\hline $36^{\prime}$ & .04328 & & .03292 & $36^{\prime}$ & .12207 & $36^{\prime}$ & .21061 \\
\hline $37^{\prime}$ & .94478 & & .03441 & $37^{\prime}$ & .12355 & $37^{\prime}$ & .21208 \\
\hline $38^{\prime}$ & .94628 & & .03500 & $38^{\prime}$ & $.125(1) 3$ & $38^{\prime}$ & .21355 \\
\hline $39^{\prime}$ & .04778 & & .03739 & $39^{\prime}$ & 2651 & $39^{\prime}$ & .21502 \\
\hline $40^{\prime}$ & $.9+927$ & & .03890 & $40^{\prime}$ & .12799 & $40^{\prime}$ & .21649 \\
\hline $4 l^{\prime}$ & .95077 & & .04037 & $41^{\prime}$ & $29+7$ & $+1^{\prime}$ & .21796 \\
\hline $42^{\prime}$ & .95227 & & $.0+186$ & $42^{\prime}$ & .13095 & $42^{\prime}$ & .21943 \\
\hline $43^{\prime}$ & .95376 & & .04335 & $43^{\prime}$ & .1 & $43^{\prime}$ & .22090 \\
\hline $44^{\prime}$ & .95526 & & .04484 & $44^{\prime}$ & .13391 & $44^{\prime}$ & .22236 \\
\hline $45^{\prime}$ & .95676 & & .09633 & $45^{\prime}$ & .13539 & $45^{\prime}$ & .22383 \\
\hline $46^{\prime}$ & .95825 & & .04781 & $46^{\prime}$ & .13687 & $46^{\prime}$ & .22530 \\
\hline $47^{\prime}$ & .95975 & & .04930 & $47^{\prime}$ & .13835 & $47^{\prime}$ & .22677 \\
\hline $48^{\prime}$ & .96124 & & .05079 & $48^{\prime}$ & .139 & $48^{\prime}$ & .22824 \\
\hline $49^{\prime}$ & .96274 & & .05228 & $+9^{\prime}$ & .14130 & $49^{\prime}$ & .22970 \\
\hline $50^{\prime}$ & .96424 & & .05377 & $50)^{\prime}$ & .14278 & $50)^{\prime}$ & .23117 \\
\hline $51^{\prime}$ & .96573 & & .05526 & $51^{\prime}$ & .14426 & $5 \mathrm{l}^{\prime}$ & .23264 \\
\hline $52^{\prime}$ & .96723 & & $.0567 \div$ & $52^{\prime}$ & .14574 & $52^{\prime}$ & .23411 \\
\hline $53^{\prime}$ & .96872 & & .05823 & $53^{\prime}$ & .14722 & $53^{\prime}$ & .23557 \\
\hline $54^{\prime}$ & .97022 & & .05972 & $54^{\prime}$ & .14870 & $5 t^{\prime}$ & .23704 \\
\hline $55^{\prime}$ & .97171 & & .06121 & $55^{\prime}$ & .15017 & $55^{\prime}$ & .23851 \\
\hline $56^{\prime}$ & .97321 & & .066239 & $56^{\prime}$ & .15165 & $56^{\prime}$ & 73997 \\
\hline $57^{\prime}$ & $.97+71$ & & .06418 & $57^{\prime}$ & .15313 & $57^{\prime}$ & $.2+144$ \\
\hline $58^{\prime}$ & .97620 & & .06567 & $58^{\prime}$ & .15461 & $58^{\prime}$ & $.2+290$ \\
\hline $59^{\prime}$ & .97770 & & .066716 & $59^{\prime}$ & .15608 & $59^{\prime}$ & .24437 \\
\hline
\end{tabular}


Table I - (continued)

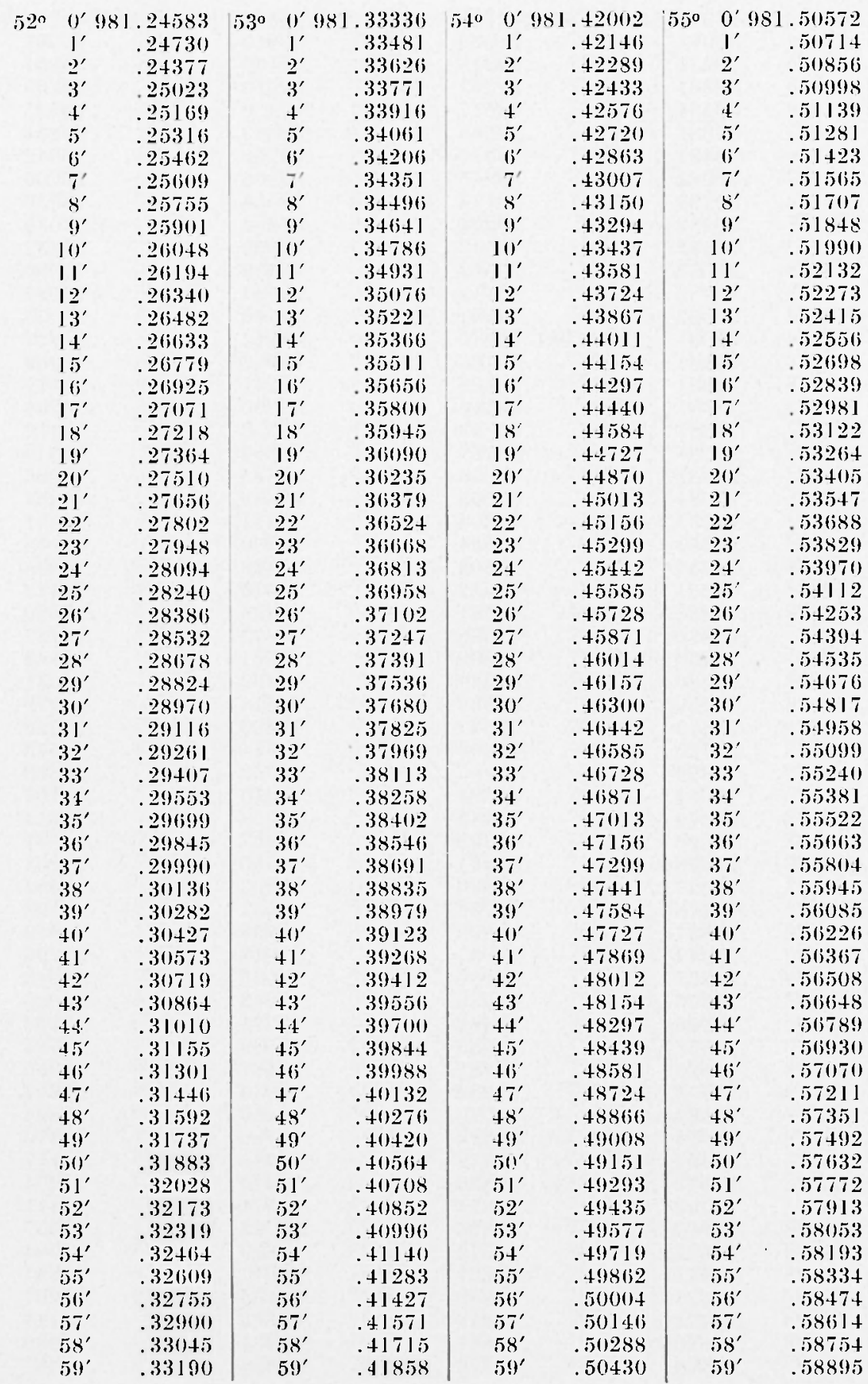


Table I - (continued)

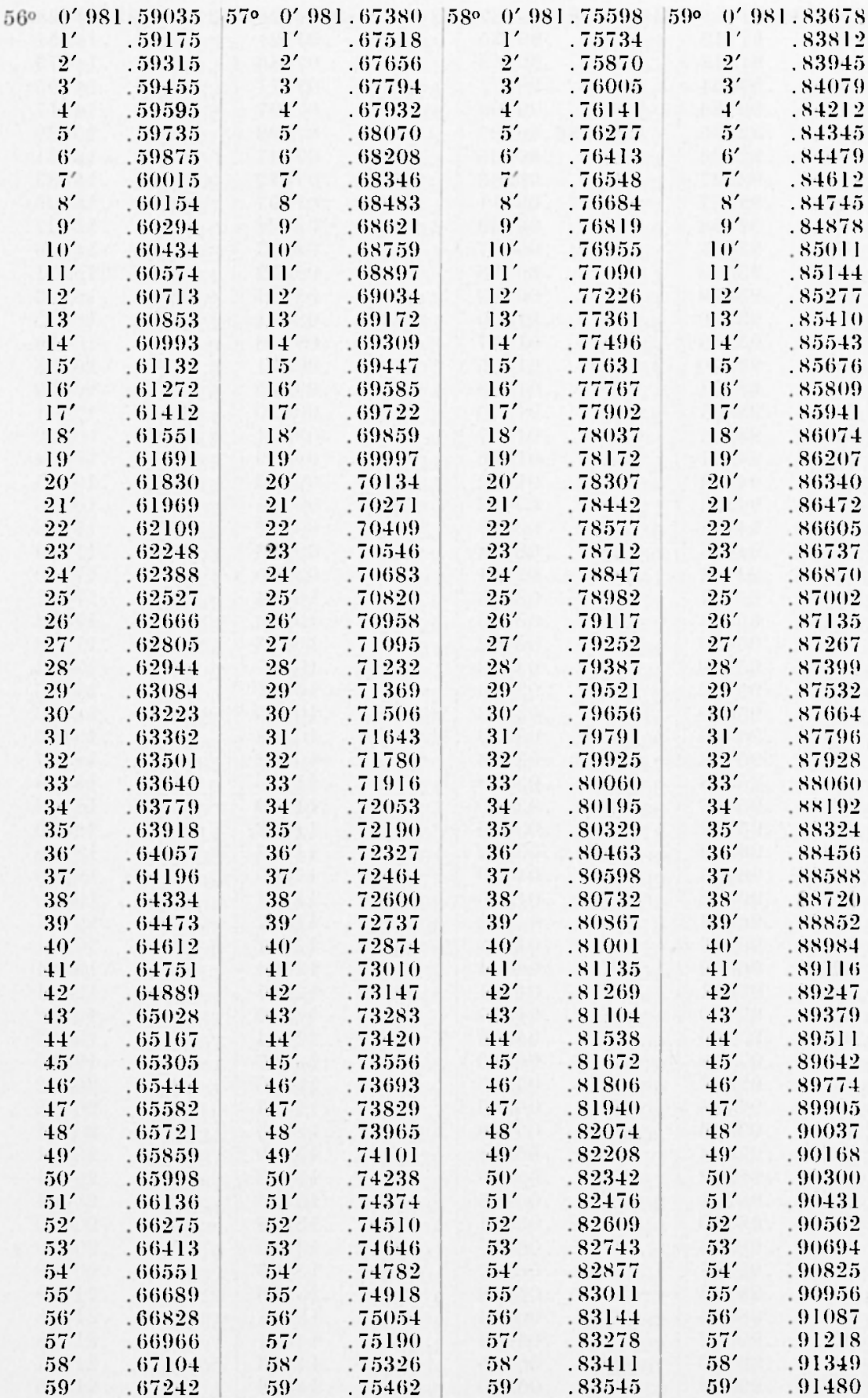


Table I - (continued)

\begin{tabular}{|c|c|c|c|c|c|c|c|c|c|c|}
\hline $0^{\prime}:$ & .91611 & 610 & & .99387 & $62^{\circ}$ & $0^{\prime}$ & .06996 & $63^{\circ}$ & $0^{\prime} 98$ & $1+428$ \\
\hline $1^{\prime}$ & .91742 & & $1^{\prime}$ & .99515 & & $I^{\prime}$ & .07121 & & $I^{\prime}$ & 14551 \\
\hline $2^{\prime}$ & .91873 & & $\mathbf{2}^{\prime}$ & .99643 & & $2^{\prime}$ & .07246 & & $2^{\prime}$ & .14673 \\
\hline $3^{\prime}$ & .02004 & & $3^{\prime}$ & .99771 & & $3^{\prime}$ & .07371 & & $3^{\prime}$ & .14795 \\
\hline $4^{\prime}$ & .92135 & & $4^{\prime}$ & .99899 & & $4^{\prime}$ & $.07+97$ & & $4^{\prime}$ & .14917 \\
\hline $5^{\prime}$ & .92265 & & $5^{\prime} 98$ & 000027 & & $5^{\prime}$ & .07622 & & $5^{\prime}$ & .15039 \\
\hline $6^{\prime}$ & .92396 & & $6^{\prime}$ & .00155 & & $6^{\prime}$ & .07747 & & $6^{\prime}$ & .15161 \\
\hline $7^{\prime}$ & .92527 & & $7^{\prime}$ & .00283 & & $7^{\prime}$ & .07872 & & $7^{\prime}$ & .15283 \\
\hline $8^{\prime}$ & .92657 & & $8^{\prime}$ & $.00+11$ & & $8^{\prime}$ & .07997 & & $\mathrm{R}^{\prime}$ & .15405 \\
\hline $9^{\prime}$ & .92788 & & $9^{\prime}$ & .00539 & & $9^{\prime}$ & .08122 & & $9^{\prime}$ & .15527 \\
\hline $10^{\prime}$ & .92918 & & I $0^{\prime}$ & .00667 & & $10^{\prime}$ & .08247 & & $0^{\prime}$ & .15649 \\
\hline $11^{\prime}$ & .93049 & & $11^{\prime}$ & .00795 & & $11^{\prime}$ & .08372 & & $I^{\prime}$ & .15771 \\
\hline $12^{\prime}$ & .93179 & & $12^{\prime}$ & .00922 & & $12^{\prime}$ & .08497 & & $2^{\prime}$ & .15893 \\
\hline $13^{\prime}$ & .93310 & & 13 & .01050 & & $13^{\prime}$ & .08621 & & $3^{\prime}$ & .16015 \\
\hline $14^{\prime}$ & .93140 & & $14^{\prime}$ & .01177 & & $14^{\prime}$ & $.087 \pm 6$ & & $4^{\prime}$ & .16136 \\
\hline $15^{\prime}$ & .93570 & & $15^{\prime}$ & .01305 & & $15^{\prime}$ & .08871 & & $5^{\prime}$ & .16258 \\
\hline $16^{\prime}$ & .93701 & & $16^{\prime}$ & . 01433 & & $16^{\prime}$ & .08995 & & $b^{\prime}$ & .16379 \\
\hline $17^{\prime}$ & .93831 & & $17^{\prime}$ & .01560 & & $17^{\prime}$ & .09120 & & $7^{\prime}$ & .16501 \\
\hline $18^{\prime}$ & .93961 & & $18^{\prime}$ & .01687 & & $18^{\prime}$ & .09244 & & $8^{\prime}$ & .16622 \\
\hline $19^{\prime}$ & .94091 & & $19^{\prime}$ & .01815 & & $19^{\prime}$ & .09369 & & $9^{\prime}$ & .16744 \\
\hline $20^{\prime}$ & .94221 & & $20^{\prime}$ & .01942 & & $20^{\prime}$ & .09493 & & $0^{\prime}$ & .16865 \\
\hline $21^{\prime}$ & .94351 & & $21^{\prime}$ & .02069 & & $2 l^{\prime}$ & .09618 & & $1^{\prime}$ & .16986 \\
\hline $22^{\prime}$ & .94481 & & $22^{\prime}$ & .02197 & & $22^{\prime}$ & .09742 & & $2^{\prime}$ & .17108 \\
\hline $23^{\prime}$ & .94611 & & $23^{\prime}$ & .02324 & & $23^{\prime}$ & .09866 & & $3^{\prime}$ & .17229 \\
\hline $24^{\prime}$ & $.947+1$ & & $24^{\prime}$ & $.02+51$ & & 2.11 & .09990 & & $4^{\prime}$ & .17350 \\
\hline $25^{\prime}$ & .94871 & & $25^{\prime}$ & .02578 & & $25^{\prime}$ & .10114 & & $5^{\prime}$ & $.17+7 \mid$ \\
\hline $26^{\prime}$ & .95001 & & $26^{\prime}$ & .02705 & & $26^{\prime}$ & .10239 & & $6^{\prime}$ & .17592 \\
\hline $27^{\prime}$ & .95130 & & $27^{\prime}$ & .02832 & & $27^{\prime}$ & .10363 & & $7^{\prime}$ & .17713 \\
\hline $28^{\prime}$ & .95260 & & $28^{\prime}$ & .02959 & & $28^{\prime}$ & .10487 & & $8^{\prime}$ & .17834 \\
\hline $29^{\prime}$ & .95390 & & $29^{\prime}$ & .03086 & & $29^{\prime}$ & .10611 & & $g^{\prime}$ & .17955 \\
\hline $30^{\prime}$ & .95519 & & $30^{\prime}$ & .03213 & & $30^{\prime}$ & .10735 & & $0^{\prime}$ & .18076 \\
\hline $31^{\prime}$ & $.956+9$ & & $31^{\prime}$ & .03339 & & $31^{\prime}$ & .10858 & & $1^{\prime}$ & .18190 \\
\hline $32^{\prime}$ & .95778 & & $32^{\prime}$ & .03466 & & $32^{\prime}$ & .10982 & & $2^{\prime}$ & .18317 \\
\hline $33^{\prime}$ & .05908 & & $33^{\prime}$ & .03593 & & $33^{\prime}$ & .11106 & & $3^{\prime}$ & .18438 \\
\hline $34^{\prime}$ & .96037 & & $34^{\prime}$ & .03720 & & $34^{\prime}$ & .11230 & & $4^{\prime}$ & 18558 \\
\hline $35^{\prime}$ & .96167 & & $35^{\prime}$ & .03846 & & $35^{\prime}$ & .11353 & & $5^{\prime}$ & .18679 \\
\hline $36^{\prime}$ & .96296 & & $36^{\prime}$ & .03973 & & $36^{\prime}$ & $.11+77$ & & $66^{\prime}$ & .18799 \\
\hline $37^{\prime}$ & .96425 & & $37^{\prime}$ & .04099 & & $37^{\prime}$ & .11600 & & $7^{\prime}$ & .18920 \\
\hline $38^{\prime}$ & .96555 & & $38^{\prime}$ & .04226 & & $38^{\prime}$ & .11724 & & $8^{\prime}$ & .19040 \\
\hline $39^{\prime}$ & .96684 & & $39^{\prime}$ & .04352 & & $39^{\prime}$ & .11847 & & $9^{\prime}$ & .19161 \\
\hline $40^{\prime}$ & .96813 & & $40^{\prime}$ & $.04+78$ & & $40^{\prime}$ & .11971 & & $0^{\prime}$ & .19281 \\
\hline $41^{\prime}$ & .96942 & & $+1^{\prime}$ & .04604 & & $+1^{\prime}$ & .12094 & & $1^{\prime}$ & .19401 \\
\hline $42^{\prime}$ & .97071 & & $42^{\prime}$ & .04731 & & $42^{\prime}$ & .12217 & & $2^{\prime}$ & .19521 \\
\hline $43^{\prime}$ & .97200 & & $43^{\prime}$ & .04857 & & $43^{\prime}$ & .12342 & & $3^{\prime}$ & .19642 \\
\hline $44^{\prime}$ & .97329 & & $44^{\prime}$ & .04983 & & $44^{\prime}$ & .12464 & & $4^{\prime}$ & .19762 \\
\hline $45^{\prime}$ & .974 .58 & & $15^{\prime}$ & .05110 & & $45^{\prime}$ & .12587 & & $5^{\prime}$ & .19882 \\
\hline $46^{\prime}$ & .97587 & & $46^{\prime}$ & .05235 & & $46^{\prime}$ & .12710 & & $6^{\prime}$ & .20002 \\
\hline $47^{\prime}$ & .97716 & & $47^{\prime}$ & .05362 & & $47^{\prime}$ & .12833 & & $7^{\prime}$ & .20122 \\
\hline $48^{\prime}$ & .97845 & & $48^{\prime}$ & .05488 & & $48^{\prime}$ & .12956 & & $18^{\prime}$ & $.202+1$ \\
\hline $49^{\prime}$ & .97974 & & $49^{\prime}$ & .05614 & & $49^{\prime}$ & .13079 & & $19^{\prime}$ & .20361 \\
\hline $50^{\prime}$ & .98102 & & $5)^{\prime}$ & .05739 & & $50^{\prime}$ & .13202 & & $30)^{\prime}$ & .20481 \\
\hline 5 & .98231 & & $51^{\prime}$ & .05865 & & $5 I^{\prime}$ & .13325 & & $51^{\prime}$ & .20601 \\
\hline $52^{\prime}$ & .98360 & & $52^{\prime}$ & .05991 & & $52^{\prime}$ & .13448 & & $52^{\prime}$ & .20720 \\
\hline $53^{\prime}$ & .98488 & & $53^{\prime}$ & .06117 & & $53^{\prime}$ & .13570 & & $53^{\prime}$ & .20840 \\
\hline $54^{\prime}$ & .98617 & & $54^{\prime}$ & .06242 & & $54^{\prime}$ & .13693 & & $4^{\prime}$ & .20959 \\
\hline $5 \overline{5}^{\prime}$ & $.987+5$ & & $5 \overline{5}^{\prime}$ & .06368 & & $55^{\prime}$ & .13816 & & $5^{\prime}$ & .21079 \\
\hline $56^{\prime}$ & .98874 & & $56^{\prime}$ & .06494 & & $56^{\prime}$ & .13938 & & $6^{\prime}$ & .21198 \\
\hline $57^{\prime}$ & .99002 & & $57^{\prime}$ & .06019 & & $57^{\prime}$ & .14061 & & $57^{\prime}$ & .21318 \\
\hline $58^{\prime}$ & .99130 & & $58^{\prime}$ & $.067+5$ & & $58^{\prime}$ & .14183 & & $8^{\prime}$ & .21437 \\
\hline $59^{\prime}$ & .99259 & & $59^{\prime}$ & .06870 & & $59^{\prime}$ & .14306 & & $69^{\prime}$ & .21556 \\
\hline
\end{tabular}


'Table I - (continuea)

\begin{tabular}{|c|c|c|c|c|c|c|c|}
\hline $64^{\circ}$ & 982.21676 & $655^{\circ}$ & 2.28729 & $66^{\circ}$ & 2.35579 & $67^{\circ}$ & 2.42218 \\
\hline$I^{\prime}$ & .21795 & $1^{\prime}$ & .28845 & $l^{\prime}$ & .356902 & $1^{\prime}$ & .42327 \\
\hline $2^{\prime}$ & .21914 & $\underline{2}^{\prime}$ & .28901 & $\underline{\underline{2}}^{\prime}$ & .35804 & $2^{\prime}$ & $.42+36$ \\
\hline $3^{\prime}$ & .22033 & $3^{\prime}$ & .29076 & $3^{\prime}$ & .35916 & $3^{\prime}$ & 42545 \\
\hline $4^{\prime}$ & .20150 & $4^{\prime}$ & .29192 & $4^{\prime}$ & $.3(j 11) 9$ & $\mathbf{t}^{\prime}$ & $42(j 53) 3$ \\
\hline $5^{\prime}$ & .22271 & $5^{\prime}$ & .20308 & $5^{\prime}$ & $.361+1$ & $\tilde{5}^{\prime}$ & 42762 \\
\hline $6^{\prime}$ & .22390 & $i^{\prime}$ & $.29+23$ & $i^{\prime}$ & .36253 & $\mathbf{6}^{\prime}$ & .42870 \\
\hline $7^{\prime}$ & $.225(19)$ & $7^{\prime}$ & .29539 & $T^{\prime}$ & .36365 & $7^{\prime}$ & .42979 \\
\hline $8^{\prime}$ & .22627 & $8^{\prime}$ & .29654 & $8^{\prime}$ & .36477 & $8^{\prime}$ & .43087 \\
\hline $9^{\prime}$ & .22746 & $9^{\prime}$ & .29770 & $9^{\prime}$ & 36589 & $9^{\prime}$ & .43196 \\
\hline $10^{\prime}$ & .22865 & $10^{\prime}$ & .29885 & $10^{\prime}$ & .36701 & $10^{\prime}$ & .43304 \\
\hline $11^{\prime}$ & .22984 & $11^{\prime}$ & $.300(100$ & $11^{\prime}$ & .36813 & $11^{\prime}$ & $43+12$ \\
\hline $12^{\prime}$ & .23102 & $12^{\prime}$ & $.3(0115$ & $12^{\prime}$ & $3(39) 24$ & $1 \underline{2}^{\prime}$ & .3520 \\
\hline $13^{\prime}$ & .23221 & $13^{\prime}$ & 30231 & $13^{\prime}$ & .37036 & $13^{\prime}$ & 43628 \\
\hline $14^{\prime}$ & .233339 & $14^{\prime}$ & .30346 & $14^{\prime}$ & .37148 & $14^{\prime}$ & .43736 \\
\hline $15^{\prime}$ & .23457 & $15^{\prime}$ & $.30+401$ & $15^{\prime}$ & .37259 & $15^{\prime}$ & .43844 \\
\hline $16^{\prime}$ & .23576 & $16^{\prime}$ & .30576 & $16^{\prime}$ & .37371 & $16^{\prime}$ & +3952 \\
\hline $17^{\prime}$ & .23694 & $17^{\prime}$ & .306991 & $17^{\prime}$ & $.37+82$ & $17^{\prime}$ & $.4+1000$ \\
\hline $18^{\prime}$ & .23812 & $18^{\prime}$ & .30800 & $18^{\prime}$ & .37594 & $18^{\prime}$ &.+4168 \\
\hline $19^{\prime}$ & .23931 & $19^{\prime}$ & .300921 & $19^{\prime}$ & $.377(15)$ & $19^{\prime}$ & .44276 \\
\hline 20$)^{\prime}$ & .24049 & $20^{\prime}$ & .31035 & $200^{\prime}$ & .37816 & $20^{\prime}$ & 44383 \\
\hline 21 & $2+167$ & $21^{\prime}$ & .31150 & 21 & 37927 & $21^{\prime}$ & $4+491$ \\
\hline $22^{\prime}$ & .24285 & $22^{\prime}$ & .31265 & $\underline{2} \mathbf{2}^{\prime}$ & .38039 & $22^{\prime}$ & .44598 \\
\hline $23^{\prime}$ & .24403 & $23^{\prime}$ & .31379 & $233^{\prime}$ & .38150 & $233^{\prime}$ & .44706 \\
\hline $24^{\prime}$ & .24521 & $24^{\prime}$ & .31494 & $24^{\prime}$ & .38261 & $24^{\prime}$ & .44813 \\
\hline $25^{\prime}$ & 24039 & $25^{\prime}$ & .31608 & 25 & 38372 & $25^{\prime}$ & +4921 \\
\hline $26^{\prime}$ & .24756 & $2 \mathbf{a}^{\prime}$ & .31723 & $26^{\prime}$ & .38483 & $26^{\prime}$ & 45028 \\
\hline $27^{\prime}$ & .24874 & $27^{\prime}$ & .31837 & $27^{\prime}$ & .38594 & $27^{\prime}$ & .45135 \\
\hline $28^{\prime}$ & $2+992$ & $28^{\prime}$ & 31951 & $28^{\prime}$ & .38704 & $28^{\prime}$ & $+52+2$ \\
\hline $299^{\prime}$ & .25109 & $29^{\prime}$ & .320106 & $29^{\prime}$ & 38815 & $29^{\prime}$ & 45334 \\
\hline $30^{\prime}$ & .25227 & $30^{\prime}$ & .32180 & $30^{\prime}$ & 38926 & $30^{\prime}$ & 45456 \\
\hline 31 & .25345 & $31^{\prime}$ & .32294 & $31^{\prime}$ & .39036 & 31 & 45563 \\
\hline $32^{\prime}$ & $.25+62$ & $32^{\prime}$ & .32408 & $32^{\prime}$ & $391+7$ & $32^{\prime}$ & .45670 \\
\hline $33^{\prime}$ & .25579 & $33^{\prime}$ & .32522 & $33^{\prime}$ & 39258 & $33^{\prime}$ & .45777 \\
\hline $34^{\prime}$ & .25697 & $34^{\prime}$ & .326366 & $34^{\prime}$ & .39368 & $34^{\prime}$ & 45884 \\
\hline $35^{\prime}$ & .25814 & $35^{\prime}$ & .32750 & $35^{\prime}$ & $.39+78$ & $35^{\prime}$ & .459991 \\
\hline $36^{\prime}$ & .25932 & $36^{\prime}$ & .32864 & $36^{\prime}$ & .39589 & $36^{\prime}$ & 46097 \\
\hline $37^{\prime}$ & .26049 & $37^{\prime}$ & .32978 & $37^{\prime}$ & 39699 & $37^{\prime}$ & 46204 \\
\hline $38^{\prime}$ & .26160 & $38^{\prime}$ & .33092 & $38^{\prime}$ & .39809 & $38^{\prime}$ & .46310 \\
\hline $39^{\prime}$ & 20283 & $39^{\prime}$ & .33205 & $39^{\prime}$ & .39919 & $39^{\prime}$ & $.46+17$ \\
\hline $40^{\prime}$ & .26400 & $40^{\prime}$ & .33319 & $40^{\prime}$ & .40029 & $40^{\prime}$ & .46523 \\
\hline $41^{\prime}$ & .26517 & $41^{\prime}$ & 33432 & $41^{\prime}$ & .40139 & $+1^{\prime}$ & $4(i 6330$ \\
\hline $42^{\prime}$ & .266334 & $42^{\prime}$ & $.335+6$ & $42^{\prime}$ & $.402+9$ & $42^{\prime}$ & 46736 \\
\hline $43^{\prime}$ & .26751 & $43^{\prime}$ & .33659 & $43^{\prime}$ & 40.359 & $13^{\prime}$ & 46842 \\
\hline $44^{\prime}$ & .26867 & $44^{\prime}$ & .33773 & $44^{\prime}$ & $.40+69$ & $44^{\prime}$ & $469+8$ \\
\hline $45^{\prime}$ & .26984 & $45^{\prime}$ & .33886 & $45^{\prime}$ & .40579 & $45^{\prime}$ & .47054 \\
\hline $46^{\prime}$ & .27101 & $4 i^{\prime}$ & .33999 & $46^{\prime}$ & .40689 & $46^{\prime}$ & .47160 \\
\hline $47^{\prime}$ & .27218 & $47^{\prime}$ & $.3+113$ & $47^{\prime}$ & .40798 & $47^{\prime}$ & .47266 \\
\hline $48^{\prime}$ & .27334 & $48^{\prime}$ & 34226 & $48^{\prime}$ & .40908 & $48^{\prime}$ & .47372 \\
\hline $49^{\prime}$ & .27451 & $49^{\prime}$ & .34339 & $49^{\prime}$ & .41017 & $49^{\prime}$ & $47+78$ \\
\hline $50^{\prime}$ & .27567 & $50^{\prime}$ & .34452 & $50^{\prime}$ & .41127 & $50^{\prime}$ & .47584 \\
\hline $5 l^{\prime}$ & .27684 & $5 \mathrm{l}^{\prime}$ & $.3+5(i 5)$ & $5 \mathrm{I}^{\prime}$ &.+1236 & $51^{\prime}$ & .476900 \\
\hline $52^{\prime}$ & .27800 & $5 \mathbf{2}^{\prime}$ & .34678 & $5 \cdot 2^{\prime}$ & $.413+1 ;$ & $52^{\prime}$ & .47795 \\
\hline $53^{\prime}$ & .27916 & $53^{\prime}$ & .34791 & $53^{\prime}$ & $.41+55$ & $53^{\prime}$ & +49001 \\
\hline $54^{\prime}$ & .28033 & $54^{\prime}$ & .34904 & $54^{\prime}$ & .41564 & $54^{\prime}$ & .48006 \\
\hline $55^{\prime}$ & .28149 & $55^{\prime}$ & .35016 & $55^{\prime}$ & 41673 & $55^{\prime}$ & .48112 \\
\hline $566^{\prime}$ & $.282(i)$ & $56^{\prime}$ & .35129 & $56^{\prime}$ &.+1783 & $566^{\prime}$ & .48217 \\
\hline $57^{\prime}$ & .28381 & 57 & .35242 & $57^{\prime}$ & .41892 & $57^{\prime}$ & .48323 \\
\hline $58^{\prime}$ & .28497 & $58^{\prime}$ & .35354 & $58^{\prime}$ & .42001 & $58^{\prime}$ & .48428 \\
\hline $59^{\prime}$ & .28613 & $59^{\prime}$ & .35467 & $59^{\prime}$ & .42110 & $59^{\prime}$ & .48533 \\
\hline
\end{tabular}


Table I - (continued)

\begin{tabular}{|c|c|c|c|c|c|c|c|}
\hline 680 & 2.48638 & $69^{\circ}$ & ' & $70^{\circ}$ & '982.60788 & $71^{0}$ & $\left(0^{\prime} 982.66504\right.$ \\
\hline $1^{\prime}$ & .48743 & & $I^{\prime} \quad .54932$ & $I^{\prime}$ & $I^{\prime} \quad .60886$ & & $l^{\prime} \quad .665997$ \\
\hline $2^{\prime}$ & 48848 & 2 & .55033 & $\mathbf{2}^{\prime}$ & .60983 & & .060090 \\
\hline $3^{\prime}$ & 48953 & & .55134 & $3^{\prime}$ & 61080 & & 66783 \\
\hline $4^{\prime}$ & .49058 & 4 & .55235 & $4^{\prime}$ & 61177 & & .66876 \\
\hline $5^{\prime}$ & .49163 & & .55336 & $5^{\prime}$ & .61274 & & .66969 \\
\hline $6^{\prime}$ & .49268 & $6^{\prime}$ & $.55+37$ & $6^{\prime}$ & 61371 & & .67062 \\
\hline $7^{\prime}$ & .49373 & & .55538 & $7^{\prime}$ & .61468 & & .67154 \\
\hline $8^{\prime}$ & .49477 & 8 & .55639 & $8^{\prime}$ & .61565 & & 67247 \\
\hline $9^{\prime}$ & .49582 & $9^{\prime}$ & .55740 & $9^{\prime}$ & .61661 & & .67340 \\
\hline $10^{\prime}$ & .49686 & $10^{\prime}$ & .55840 & $10^{\prime}$ & .61758 & 10 & $67+32$ \\
\hline $11^{\prime}$ & .49791 & $11^{\prime}$ & I.5941 & $1 I^{\prime}$ & .61855 & II & .67525 \\
\hline $12^{\prime}$ & .49895 & 12 & .56041 & $12^{\prime}$ & .61951 & 12 & .67617 \\
\hline $13^{\prime}$ & .50000 & 13 & .56142 & $13^{\prime}$ & .62047 & 13 & .67709 \\
\hline $14^{\prime}$ & .50104 & $14^{\prime}$ & .56242 & $14^{\prime}$ & .62144 & 14 & .67802 \\
\hline $15^{\prime}$ & .50208 & 15 & .56342 & $15^{\prime}$ & .62240 & 15 & 67894 \\
\hline $16^{\prime}$ & .50312 & $16^{\prime}$ & .56443 & $16^{\prime}$ & .62336 & 16 & 67986 \\
\hline $17^{\prime}$ & .50416 & $17^{\prime}$ & .56543 & $17^{\prime}$ & .62433 & 17 & .68078 \\
\hline $18^{\prime}$ & .50520 & 18 & .566643 & $18^{\prime}$ & .62529 & 18 & 68170 \\
\hline $19^{\prime}$ & .50694 & $19^{\prime}$ & .56743 & $19^{\prime}$ & .62625 & 19 & .68262 \\
\hline 20$)^{\prime}$ & .50728 & $20^{\prime}$ & .56843 & $20^{\prime}$ & .62721 & 20 & .68354 \\
\hline $2 I^{\prime}$ & .50832 & $21^{\prime}$ & .56943 & $2 I^{\prime}$ & .62817 & 21 & $68+46$ \\
\hline $22^{\prime}$ & 50936 & $22^{\prime}$ & .57043 & $22^{\prime}$ & (62912 & 22 & 68537 \\
\hline $23^{\prime}$ & 51039 & 23 & $.57 / 43$ & $23^{\prime}$ & .03008 & 23 & 68629 \\
\hline $24^{\prime}$ & .51143 & 24 & .57242 & $24^{\prime}$ & .63104 & 24 & .68720 \\
\hline $25^{\prime}$ & .51247 & $25^{\prime}$ & .57342 & $25^{\prime}$ & .63200 & 25 & 68812 \\
\hline $26^{\prime}$ & .51350 & $26^{\prime}$ & .57442 & $26^{\prime}$ & . (33295 & 26 & 68903 \\
\hline $27^{\prime}$ & 51453 & 27 & .57541 & $27^{\prime}$ & . (33391 & 27 & 68995 \\
\hline $28^{\prime}$ & .51557 & 28 & $.576+1$ & $28^{\prime}$ & 63486 & 28 & .69086 \\
\hline $29^{\prime}$ & .51660 & $29^{\prime}$ & 57740 & $29^{\prime}$ & .63581 & 29 & 69177 \\
\hline $30^{\prime}$ & .51763 & $30^{\prime}$ & .57839 & $30^{\prime}$ & .63677 & 30 & 69268 \\
\hline $31^{\prime}$ & .51867 & $31^{\prime}$ & 57939 & $31^{\prime}$ & 63772 & 31 & 69359 \\
\hline $32^{\prime}$ & .51970 & $32^{\prime}$ & .58038 & $32^{\prime}$ & .03867 & 32 & .69450 \\
\hline $33^{\prime}$ & .52023 & 33 & .58137 & $33^{\prime}$ & 63962 & 33 & 69541 \\
\hline $34^{\prime}$ & .52176 & $34^{\prime}$ & .58236 & $34^{\prime}$ & .64057 & 34 & 69632 \\
\hline $35^{\prime}$ & .52279 & $35^{\prime}$ & .58335 & $35^{\prime}$ & $6+152$ & 35 & .69723 \\
\hline $36^{\prime}$ & .52382 & $36^{\prime}$ & .584 & $36^{\prime}$ & .64247 & 36 & .69814 \\
\hline $37^{\prime}$ & .52484 & $37^{\prime}$ & .58533 & $37^{\prime}$ & .64342 & 37 & 69904 \\
\hline $38^{\prime}$ & .52587 & $38^{\prime}$ & .586 & $38^{\prime}$ & .64437 & 38 & 69995 \\
\hline $39^{\prime}$ & 52690 & $39^{\prime}$ & .58730 & $39^{\prime}$ & $6+531$ & 39 & .70085 \\
\hline $40^{\prime}$ & .52792 & $40^{\prime}$ & .588 & $40^{\prime}$ & .64626 & 40 & .70176 \\
\hline $41^{\prime}$ & 52895 & $+1^{\prime}$ & .58928 & $41^{\prime}$ & .64720 & 41 & 70266 \\
\hline $42^{\prime}$ & .52997 & $42^{\prime}$ & .59026 & $42^{\prime}$ & .64815 & 42 & .70357 \\
\hline $43^{\prime}$ & .53100 & $43^{\prime}$ & .59125 & $43^{\prime}$ & 64909 & 43 & .70447 \\
\hline $44^{\prime}$ & $.532(02$ & $44^{\prime}$ & .59223 & $44^{\prime}$ & .65004 & 44 & .70537 \\
\hline $45^{\prime}$ & .53304 & $45^{\prime}$ & .59321 & $45^{\prime}$ & 65098 & 45 & .70627 \\
\hline $46^{\prime}$ & .53407 & $46^{\prime}$ & .59420 & $46^{\prime}$ & 651 & 46 & דודסד. \\
\hline $47^{\prime}$ & .53509 & $47^{\prime}$ & .59518 & $47^{\prime}$ & .65286 & 47 & ד 7080 \\
\hline $48^{\prime}$ & .53611 & $48^{\prime}$ & .59616 & $48^{\prime}$ & .65380 & 48 & 70897 \\
\hline $49^{\prime}$ & .53713 & $49^{\prime}$ & .59714 & $49^{\prime}$ & .65474 & 49 & 70987 \\
\hline $50^{\prime}$ & 53815 & 50 & .59812 & $50^{\prime}$ & 65568 & 50 & .71076 \\
\hline $51^{\prime}$ & .53917 & 51 & .59910 & $5 \mathrm{l}^{\prime}$ & .65662 & 51 & 71166 \\
\hline $52^{\prime}$ & .54019 & 52 & .60008 & $52^{\prime}$ & .65756 & 52 & 71250 \\
\hline $53^{\prime}$ & .54120 & 53 & .60106 & $53^{\prime}$ & 65850 & 53 & .71345 \\
\hline $54^{\prime}$ & .54222 & 54 & .60203 & $54^{\prime}$ & .65943 & 54 & .71435 \\
\hline $55^{\prime}$ & .54324 & 55 & .60301 & $55^{\prime}$ & 66037 & 55 & .71524 \\
\hline $56^{\prime}$ & .54425 & 56 & 60399 & $56^{\prime}$ & 66130 & 56 & .71613 \\
\hline $57^{\prime}$ & .54527 & 57 & .60496 & $57^{\prime}$ & .66224 & 57 & .71703 \\
\hline $58^{\prime}$ & .54628 & 58 & 60594 & $58^{\prime}$ & .66317 & 58 & .71792 \\
\hline $59^{\prime}$ & .54729 & 09 & .60691 & $59^{\prime}$ & $66+10$ & 59 & .71881 \\
\hline
\end{tabular}


Table I - (continued)

\begin{tabular}{|c|c|c|c|c|c|c|c|c|}
\hline $0^{\prime}$ & .71970 & $73^{\circ}$ & $0^{\prime} !$ & .77180 & $74^{\circ}$ & 2.82127 & $75^{\circ}$ & 2.86807 \\
\hline$I^{\prime}$ & .72059 & & $1^{\prime}$ & .77264 & $I^{\prime}$ & .82208 & $I^{\prime}$ & $.86 \sin 2$ \\
\hline $2^{\prime}$ & .72148 & & $\underline{2}^{\prime}$ & .77349 & $2^{\prime}$ & .82288 & $2^{\prime}$ & .86958 \\
\hline $3^{\prime}$ & .72236 & & $3^{\prime}$ & $.77+34$ & $3^{\prime}$ &.$\$ 2368$ & $3^{\prime}$ & .87033 \\
\hline $4^{\prime}$ & .72325 & & $4^{\prime}$ & .77518 & $4^{\prime}$ & $82+48$ & $4^{\prime}$ & S7109 \\
\hline $5^{\prime}$ & .72414 & & $\bar{y}^{\prime}$ & 776010 & $\overline{5}^{\prime}$ & .82528 & 5 & .87184 \\
\hline $6^{\prime}$ & .72502 & & $i^{\prime}$ & .77687 & $6^{\prime}$ & .826018 & $i^{\prime}$ & .87260 \\
\hline $7^{\prime}$ & .72591 & & $7^{\prime}$ & I & $7^{\prime}$ & .82687 & $7^{\prime}$ & .87335 \\
\hline $8^{\prime}$ & .72679 & & $8^{\prime}$ & .77855 & $8^{\prime}$ & $.8276 s$ & $8^{\prime}$ & $.87+10$ \\
\hline $\boldsymbol{\theta}^{\prime}$ & .72768 & & $9^{\prime}$ & .77939 & $g^{\prime}$ & .82847 & $9^{\prime}$ & $.87+85$ \\
\hline $10^{\prime}$ & .72856 & & $10^{\prime}$ & .78023 & $10^{\prime}$ & .82926 & $100^{\prime}$ & .87560 \\
\hline $11^{\prime}$ & $729+4$ & & $11^{\prime}$ & .78107 & $\mathrm{II}^{\circ}$ & .83006 & $\mathrm{II}^{\prime}$ & .876335 \\
\hline $12^{\prime}$ & .73032 & & $12^{\prime}$ & 78191 & $12^{\prime}$ & .83085 & $12^{\prime}$ & .87710 \\
\hline $13^{\prime}$ & .73121 & & $13^{\prime}$ & .78274 & $13^{\prime}$ & .83164 & $13^{\prime}$ & .87784 \\
\hline $1 t^{\prime}$ & .73209 & & $14^{\prime}$ & .78358 & $14^{\prime}$ & .83244 & $14^{\prime}$ & .87859 \\
\hline $15^{\prime}$ & .73297 & & $15^{\prime}$ & .78442 & $155^{\prime}$ & .83323 & $1 \bar{s}^{\prime}$ & .87934 \\
\hline $16^{\prime}$ & .73384 & & $16^{\prime}$ & .78525 & $16^{\prime}$ & .834112 & $16^{\prime}$ & .88000 \\
\hline $17^{\prime}$ & $.73+72$ & & $17^{\prime}$ & .78609 & 17 & .83 .41 & $17^{\prime}$ & .85083 \\
\hline $18^{\prime}$ & .73560 & & $18^{\prime}$ & .786902 & $18^{\prime}$ & .83560 & $18^{\prime}$ & 88157 \\
\hline $19^{\prime}$ & .73648 & & $19^{\prime}$ & .78775 & $19^{\prime}$ & .83639 & $19^{\prime}$ & 88231 \\
\hline $20^{\prime}$ & .73735 & & $20^{\prime}$ & .78859 & $20^{\prime}$ & .83717 & 2()$^{\prime}$ & 88306 \\
\hline $2 \mathrm{l}^{\prime}$ & .73823 & & $21^{\prime}$ & 78942 & $21^{\prime}$ & .83790 & $2]^{\prime}$ & .88380 \\
\hline $22^{\prime}$ & .73910 & & $22^{\prime}$ & .79025 & $22^{\prime}$ & .83875 & $22^{\prime}$ & .88454 \\
\hline $23^{\prime}$ & $.7399 \mathrm{~s}$ & & $23^{\prime}$ & .79108 & $23^{\prime}$ & .83953 & $23^{\prime}$ & .88528 \\
\hline $24^{\prime}$ & .74085 & & $24^{\prime}$ & .79191 & $24^{\prime}$ & .84032 & $24^{\prime}$ & sstiole \\
\hline $25^{\prime}$ & $7+172$ & & $25^{\prime}$ & .79274 & $25^{\prime}$ & .84110 & $25^{\prime}$ & .88676 \\
\hline $26^{\prime}$ & 74259 & & $26^{\prime}$ & .79356 & $266^{\prime}$ & .84188 & $26^{\prime}$ & .88749 \\
\hline $27^{\prime}$ & $.7+346$ & & $27^{\prime}$ & 79439 & $27^{\prime}$ & .84267 & $27^{\prime}$ & 88823 \\
\hline $28^{\prime}$ & .74433 & & $28^{\prime}$ & 79522 & $28^{\prime}$ & .84345 & $28^{\prime}$ & 88897 \\
\hline $29^{\prime}$ & 74520 & & $29^{\prime}$ & .79604 & $29^{\prime}$ & .84423 & $29^{\prime}$ & . 8 so70 \\
\hline $30^{\prime}$ & 74607 & & $30^{\prime}$ & 79687 & $30^{\prime}$ & .84501 & $3 v^{\prime}$ & $890+4$ \\
\hline $31^{\prime}$ & 74694 & & $31^{\prime}$ & 79769 & $31^{\circ}$ & 84579 & $3 l^{\prime}$ & \\
\hline $3 \cdot 2^{\prime}$ & .74781 & & $32^{\prime}$ & 79852 & $32^{\prime}$ & 84657 & $32^{\prime}$ & 89190 \\
\hline $33^{\prime}$ & .74867 & & $33^{\prime}$ & 79934 & $33^{\prime}$ & .84735 & $33^{\prime}$ & .89264 \\
\hline $34^{\prime}$ & .74954 & & $34^{\prime}$ & 80016 & $34^{\prime}$ & .84812 & $34^{\prime}$ & .89337 \\
\hline $35^{\prime}$ & .75()$+1$ & & $35^{\prime}$ & .80098 & $35^{\prime}$ & 84890 & $35^{\prime}$ & $89+10$ \\
\hline $36^{\prime}$ & 75127 & & $36^{\prime}$ & .80180 & $36^{\prime}$ & .84968 & $36^{\prime}$ & $.89+83$ \\
\hline $37^{\prime}$ & .75213 & & $37^{\prime}$ & .80262 & $37^{\prime}$ & $.850+5$ & $37^{\prime}$ & .89556 \\
\hline $38^{\prime}$ & .75300 & & $38^{\prime}$ & .80344 & $38^{\prime}$ & .85122 & $38^{\prime}$ & $89(629)$ \\
\hline $39^{\prime}$ & .75386 & & $39^{\prime}$ & $.80+26$ & $39^{\prime}$ & .85200 & $39^{\prime}$ & . \\
\hline $40^{\prime}$ & .75472 & & $40^{\prime}$ & .80508 & +()$^{\prime}$ & .85277 & 4()$^{\prime}$ & 89774 \\
\hline $41^{\prime}$ & . & & $4 I^{\prime}$ & .80590 & $41^{\prime}$ & .85354 & $+1^{\prime}$ & $.898+7$ \\
\hline $42^{\prime}$ & .75644 & & $+2^{\prime}$ & .80671 & $+2^{\prime}$ & .85431 & $+2^{\prime}$ & 89919 \\
\hline $43^{\prime}$ & .75730 & & $43^{\prime}$ & .80753 & $+3^{\prime}$ & 85508 & $43^{\prime}$ & .89992 \\
\hline $44^{\prime}$ & .75816 & & $44^{\prime}$ & .80834 & $44^{\prime}$ & . 85585 & $44^{\prime}$ & .90004 \\
\hline $45^{\prime}$ & .75902 & & $45^{\prime}$ & .80916 & $45^{\prime}$ & .85662 & $45^{\prime}$ & .901336 \\
\hline $46^{\prime}$ & 75987 & & $46^{\prime}$ & 80997 & $460^{\prime}$ & .85739 & $46^{\prime}$ & .9010208 \\
\hline $47^{\prime}$ & .76073 & & $47^{\prime}$ & .81078 & $47^{\prime}$ & .85816 & $+7^{\prime}$ & $.0(1281$ \\
\hline $48^{\prime}$ & .76159 & & $48^{\prime}$ & .81159 & $48^{\prime}$ & .85893 & $48^{\prime}$ & .001353 \\
\hline $49^{\prime}$ & 76244 & & $49^{\prime}$ & 81240 & $49^{\prime}$ & .85969 & $49^{\prime}$ & $.90+25$ \\
\hline $50^{\prime}$ & .76330 & & $50^{\prime}$ & .81321 & $50^{\prime}$ & .86046 & . & .90497 \\
\hline $5 l^{\prime}$ & .76415 & & $5 I^{\prime}$ & .81402 & $5 \mathrm{l}^{\prime}$ & .861122 & $5 l^{\prime}$ & 90568 \\
\hline $52^{\prime}$ & 76500 & & $52^{\prime}$ & .81483 & $52^{\prime}$ & .86198 & $52^{\prime}$ & $.90(i+0)$ \\
\hline $53^{\prime}$ & .76585 & & $53^{\prime}$ & .81564 & $53^{\prime}$ & 860275 & $53^{\prime}$ & .90712 \\
\hline $54^{\prime}$ & 76671 & & $54^{\prime}$ & $8 \perp 645$ & $54^{\prime}$ & .86351 & $54^{\prime}$ & .90783 \\
\hline $55^{\prime}$ & .76756 & & $55^{\prime}$ & .81725 & $55^{\prime}$ & .86427 & $55^{\prime}$ & .90855 \\
\hline $56^{\prime}$ & 76841 & & $56^{\prime}$ & .81806 & $56^{\prime}$ & 865013 & $56^{\prime}$ & .90926 \\
\hline $57^{\prime}$ & .76926 & & $57^{\prime}$ & .81886 & $57^{\prime}$ & .86579 & $57^{\prime}$ & \\
\hline $5 s^{\prime}$ & .77010 & & $58^{\prime}$ & .81967 & $58^{\prime}$ & .86655 & $58^{\prime}$ & .91060 \\
\hline $59^{\prime}$ & .77095 & & $59^{\prime}$ & 82047 & $59^{\prime}$ & .86731 & $59^{\prime}$ & .91140 \\
\hline
\end{tabular}


Fable I - (continued)

\begin{tabular}{|c|c|c|c|c|c|c|c|}
\hline $76^{\circ}$ & 982.91211 & $77^{\circ}$ & 2.95337 & $78^{\circ}$ & $00^{\prime} 982.99177$ & 790 & 3.02728 \\
\hline$I^{\prime}$ & .91283 & $1^{\prime}$ & .95403 & & $1^{\prime} \quad .99238$ & $1^{\prime}$ & .02784 \\
\hline $2^{\prime}$ & .91353 & $2^{\prime}$ & .95469 & & .993000 & $2^{\prime}$ & .02841 \\
\hline $3^{\prime}$ & 91 424 & $3^{\prime}$ & .95535 & & .99361 & $3^{\prime}$ & .02898 \\
\hline $4^{\prime}$ & .91495 & $4^{\prime}$ & .95602 & & $.99+23$ & $4^{\prime}$ & .02954 \\
\hline $5^{\prime}$ & .91566 & $5^{\prime}$ & .95668 & & .99484 & $5^{\prime}$ & .03010 \\
\hline $66^{\prime}$ & .91637 & $6^{\prime}$ & .95734 & & .99545 & $6 j^{\prime}$ & .03067 \\
\hline $7^{\prime}$ & .91707 & $7^{\prime}$ & .95799 & & .99606 & $7^{\prime}$ & .03123 \\
\hline $8^{\prime}$ & .91778 & $8^{\prime}$ & .95865 & & .996667 & $8^{\prime}$ & .03179 \\
\hline $9^{\prime}$ & .91848 & $9^{\prime}$ & .95931 & & .99728 & $9^{\prime}$ & .03235 \\
\hline $10^{\prime}$ & .91919 & $10^{\prime}$ & .95097 & & .99789 & $10^{\prime}$ & .03291 \\
\hline $11^{\prime}$ & .91989 & $11^{\prime}$ & .96062 & & .99850 & $11^{\prime}$ & $.033+7$ \\
\hline $12^{\prime}$ & .92059 & $12^{\prime}$ & .96128 & & .99910 & $12^{\prime}$ & .03403 \\
\hline $13^{\prime}$ & .92129 & $13^{\prime}$ & .96193 & & .99971 & $13^{\prime}$ & $.03+58$ \\
\hline $14^{\prime}$ & .92199 & $14^{\prime}$ & .96258 & & $t^{\prime} 983.00031$ & $14^{\prime}$ & .03514 \\
\hline $15^{\prime}$ & .92269 & $15^{\prime}$ & .96324 & & $5^{\prime} \quad .00092$ & $15^{\prime}$ & .03570 \\
\hline $16^{\prime}$ & .92339 & $16^{\prime}$ & .96389 & & .00152 & $16^{\prime}$ & .03625 \\
\hline $17^{\prime}$ & .92409 & $17^{\prime}$ & .96454 & & .00213 & $17^{\prime}$ & .03681 \\
\hline $18^{\prime}$ & .92479 & $18^{\prime}$ & .96519 & & .00273 & $18^{\prime}$ & 0.03736 \\
\hline $19^{\prime}$ & .92548 & $19^{\prime}$ & .96584 & & .00333 & $19^{\prime}$ & .03791 \\
\hline $20^{\prime}$ & .92618 & $20^{\prime}$ & .96649 & & .00393 & $20^{\prime}$ & .03846 \\
\hline $21^{\prime}$ & .92687 & $21^{\prime}$ & .96713 & & .00453 & $2 l^{\prime}$ & .03901 \\
\hline $22^{\prime}$ & .02757 & $22^{\prime}$ & .96778 & & .00513 & $22^{\prime}$ & .03956 \\
\hline $23^{\prime}$ & .92826 & $23^{\prime}$ & .96843 & & .00573 & $23^{\prime}$ & .04011 \\
\hline $24^{\prime}$ & .92895 & $24^{\prime}$ & .96907 & & .00632 & $24^{\prime}$ & $.04060 \mathrm{j}$ \\
\hline $25^{\prime}$ & .92965 & $25^{\prime}$ & .96972 & & .00692 & $25^{\prime}$ & .04121 \\
\hline $26^{\prime}$ & .93034 & $26^{\prime}$ & .97036 & & 00751 & $26^{\prime}$ & .04175 \\
\hline $27^{\prime}$ & .93103 & $27^{\prime}$ & .97100 & & .00811 & 27 & .04230 \\
\hline $28^{\prime}$ & .93172 & $28^{\prime}$ & .97164 & & .00870 & $28^{\prime}$ & .04284 \\
\hline $29^{\prime}$ & .93241 & $29^{\prime}$ & .97229 & & .00930 & $29^{\prime}$ & .04339 \\
\hline $30^{\prime}$ & .93309 & $30^{\prime}$ & .97293 & & .00989 & $30)^{\prime}$ & .04393 \\
\hline $31^{\prime}$ & .93378 & $31^{\prime}$ & .97357 & & (0) 048 & $31^{\prime}$ & (1) 4447 \\
\hline $32^{\prime}$ & .93447 & $32^{\prime}$ & $.97+21$ & & .01107 & $32^{\prime}$ & .04502 \\
\hline $33^{\prime}$ & .93515 & $33^{\prime}$ & .97484 & & .01160 & $33^{\prime}$ & .04550 \\
\hline $34^{\prime}$ & .93584 & $34^{\prime}$ & .97548 & & .01225 & $34^{\prime}$ & $.04(j] 0$ \\
\hline $35^{\prime}$ & .93652 & $35^{\prime}$ & .97612 & & .01284 & $355^{\prime}$ & 04664 \\
\hline $36 i^{\prime}$ & .93720 & $36^{\prime}$ & .97675 & & (0) 342 & $36^{\prime}$ & .04717 \\
\hline $37^{\prime}$ & .93789 & $37^{\prime}$ & .97739 & & .01401 & $37^{\prime}$ & .04771 \\
\hline $38^{\prime}$ & .93857 & $38^{\prime}$ & .97802 & & .01460 & $38^{\prime}$ & .04825 \\
\hline $39^{\prime}$ & .93925 & $39^{\prime}$ & .97865 & & .01518 & $39^{\prime}$ & .04878 \\
\hline $40^{\prime}$ & .93993 & $40)^{\prime}$ & .97929 & & (1)1577 & $40^{\prime}$ & 0.4932 \\
\hline $41^{\prime}$ & .94061 & $41^{\prime}$ & .97992 & & .01635 & $41^{\prime}$ & .04985 \\
\hline $42^{\prime}$ & .94129 & $42^{\prime}$ & .98055 & & .01693 & $42^{\prime}$ & .05039 \\
\hline $43^{\prime}$ & .94196 & $43^{\prime}$ & .98118 & & (0) 1501 & $43^{\prime}$ & .05092 \\
\hline $44^{\prime}$ & .94264 & $44^{\prime}$ & .98181 & & .01809 & $+4^{\prime}$ & (0) 145 \\
\hline $45^{\prime}$ & .94332 & $45^{\prime}$ & .98244 & & .01867 & $45^{\prime}$ & .05198 \\
\hline $46^{\prime}$ & .94399 & $46^{\prime}$ & .98307 & & .01925 & $46^{\prime}$ & .05251 \\
\hline $47^{\prime}$ & $.94+67$ & $47^{\prime}$ & .98369 & & .01983 & $47^{\prime}$ & .053304 \\
\hline $48^{\prime}$ & .94534 & $48^{\prime}$ & $.98+32$ & & $.0204]$ & $48^{\prime}$ & .05357 \\
\hline $49^{\prime}$ & $.9+601$ & $49^{\prime}$ & $.98+94$ & & .02090 & $49^{\prime}$ & $.05+10$ \\
\hline $50^{\prime}$ & $.9+669$ & $500^{\prime}$ & .98557 & & .02156 & 5()$^{\prime}$ & $.05+62$ \\
\hline $51^{\prime}$ & .94736 & $51^{\prime}$ & .98619 & & .02214 & $5]^{\prime}$ & .05515 \\
\hline $52^{\prime}$ & .94803 & $52^{\prime}$ & .98681 & & .02271 & $52^{\prime}$ & .055067 \\
\hline $53^{\prime}$ & .94870 & $53^{\prime}$ & $.9874+$ & & .02329 & $53^{\prime}$ & $.05(\mathrm{i} 20$ \\
\hline $54^{\prime}$ & .94937 & $54^{\prime}$ & .98806 & & .02386 & $54^{\prime}$ & .05672 \\
\hline $55^{\prime}$ & .95004 & $55^{\prime}$ & .98868 & & $.024+3$ & $55^{\prime}$ & 0,5724 \\
\hline $566^{\prime}$ & .95070 & $56^{\prime}$ & .98930 & & .02500 & $56^{\prime}$ & .05777 \\
\hline $57^{\prime}$ & .95137 & $57^{\prime}$ & .98992 & & .02557 & $57^{\prime}$ & (1)5829 \\
\hline $58^{\prime}$ & .952014 & $58^{\prime}$ & .09054 & & $.026] 4$ & $58^{\prime}$ & .05881 \\
\hline $59^{\prime}$ & .95270 & $59^{\prime}$ & 5911 & & .02671 & $59^{\prime}$ & .05933 \\
\hline
\end{tabular}


Table I - (continued)

$8\left(1^{\circ}\right.$
0.05985

$I^{\prime} \quad .06036$

$2^{\prime} \quad .06088$

$\left.3^{\prime} .06 \mid+1\right)$

$4^{\prime} \quad .06191$

$5^{\prime} \quad .06243$

$6^{\prime} \quad .06294$

$7^{\prime} \quad 0(3345$

$8^{\prime} \quad .06396$

$9^{\prime} \quad .06448$

$101^{\prime} \quad .06499$

$11^{\prime} .065+9$

$12^{\prime} \quad .066000$

$13^{\prime} \quad 06(551$

$14^{\prime} \quad .06702$

$15^{\prime} \quad .06752$

I $6^{\prime} \quad .06803$

$17^{\prime} \quad .06853$

$18^{\prime} \quad .06904$

$19^{\prime} \quad .06954$

$20)^{\prime} \quad 07004$

$21^{\prime} \quad .07054$

$2 \cdot 2^{\prime} \quad .07104$

$23^{\prime} .07154$

$24^{\prime} \quad .07204$

$25^{\prime} \quad 07254$

$266^{\prime} \quad .07304$

$27^{\prime} \quad .07353$

$28, \quad 07+03$

$29^{\prime} \quad .17+52$

$30 . \quad 07502$

$31^{\prime} .07551$

$3: 2^{\prime} \quad .076(\%)$

$33^{\prime} \quad 07649$

$34^{\prime} \quad .07698$

$35^{\prime} \quad 07747$

$36^{\prime} \quad .07796$

$37^{\prime} \quad .07845$

$38^{\prime} \quad .07893$

$39^{\prime} \quad .07942$

$40)^{\prime} \quad .0799 \mathrm{~J}$

$+1^{\prime}$. os 039

$42^{\prime} \quad .08087$

$43^{\prime} \quad 08136$

$44^{\prime} \quad .08184$

$4)^{\prime} \quad .08232$

$46^{\prime} \quad .08280$

$47^{\prime} \quad .08328$

$48^{\prime} \quad .08376$

$49^{\prime} \quad .08+24$

$500^{\prime} .08471$

$51^{\prime} .08519$

$52^{\prime} \quad .08566$

$53^{\prime} .08614$

$54^{\prime}$.

$55^{\prime} .08708$

$56^{\prime} \quad .18756$

$57^{\prime} .08803$

$58^{\prime} .08850$

$59^{\prime} \quad .118897$ $81^{\circ} \quad 0^{\prime} 983.08944$

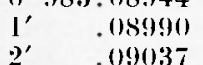

$\begin{array}{ll}2^{\prime} & .090137 \\ 3^{\prime} & .090(084\end{array}$

$4^{\prime} \quad .09130$

$5^{\prime} \quad .09177$

$6^{\prime} \quad .09223$

$7^{\prime} \quad .099269$

$8^{\prime} \quad .09315$

$9^{\prime} \quad .09361$

$10^{\prime} \quad .09407$

$1 I^{\prime} \quad .09453$

$12^{\prime}$

$13^{\prime}$

$14^{\prime}$

$15^{\prime}$

$16^{\prime}$

$17^{\prime}$

$18^{\prime}$

$19^{\prime}$

$20^{\prime}$

$2 \mathrm{I}^{\prime}$

$22^{\prime}$

$24^{\prime}$

$25^{\prime}$
$266^{\prime}$

$26^{\prime}$

$28^{\prime}$

$29^{\prime}$

$30^{\prime}$

$3 I^{\prime}$

$32^{\prime}$

$33^{\prime}$

$3 t^{\prime}$

$36^{\prime}$

$37^{\prime}$

$38^{\prime}$

$39^{\prime}$

$4)^{\prime}$

$41^{\prime}$

$42^{\prime}$

$43^{\prime}$

$44^{\prime}$

$45^{\prime}$

$46^{\prime}$

$47^{\prime}$

$48^{\prime}$

$49^{\prime}$

$50^{\prime}$

$5 I^{\prime}$

$52^{\prime}$

$53^{\prime}$

$54^{\prime}$

$55^{\prime}$

$56^{\prime}$

57

$58^{\prime}$
.09499

.09545

.09591

$.09636 \mathrm{j}$

.09682

.09727

.09773

.09818

.09863

.09908

.09953

.09998

.10043

.10088

. 10132

.10177

.10221

.10266

.10310

.10354

. 10399

.10443

.10487

.10531

.10574

.10618

.10662

.10705

$.107+9$

.10792

.10836

.10879

.10922

$.109(6) 5$

.11008

. 11051

. 11094

.11136

.11179

. 11222

. 1 264

.11307

.11349

.11391

.11433

.11475

.11517

.11559
$820 \quad 0,983.11601$ I' 11643

$2^{\prime} .11684$

$3^{\prime} .11726$

$4^{\prime} .11767$

$5^{\prime} .11809$

$6^{\prime} .11850$

$7^{\prime} .11891$

$8^{\prime} \quad .11932$

$9^{\prime} .11973$

$100^{\prime} .12014$

$1 \mathrm{I}^{\prime} \quad .12055$

$12^{\prime} \quad .12096$

$13^{\prime} \quad .12137$

$14^{\prime} .12177$

$15)^{\prime} .12218$

$16^{\prime} \quad .12258$

$17^{\prime} \quad .12299$

$18^{\prime} \quad .12339$

$19^{\prime} \quad .12379$

$20^{\prime} \quad .12+19$

$21^{\prime} \quad .12459$

$22^{\prime} \quad .12499$

$23^{\prime} \quad .12539$

$24^{\prime} \quad .12579$

$25^{\prime} \quad .12618$

$26^{\prime} \quad .12658$

$27^{\prime} \quad .12697$

$28^{\prime} \quad .12737$

$29^{\prime} \quad .12776$

$30^{\prime} .12815$

$31^{\prime} \quad .12855$

$32^{\prime} \quad .12894$

$33^{\prime} \quad .12933$

$34^{\prime} \quad .12972$

$35^{\prime} .13010$

$36 j^{\prime} \quad .13049$

$37^{\prime}$. 13088

$38^{\prime} .13126$

$39^{\prime} \quad .131(55)$

$40)^{\prime} \quad .132(1) 3$

$41^{\prime} \quad .13242$

$42^{\prime} .13280^{\prime}$

$+3^{\prime} .13318$

$44^{\prime} \quad .13350$

$45^{\prime} \quad .13394$

$46^{\prime} \quad .13432$

$47^{\prime} \quad .13470$

$45^{\prime} \quad .13507$

$49^{\prime} \quad .13545$

$500^{\prime} \quad .13583$

$51^{\prime} \quad .13620$

$52^{\prime} \quad .13657$

$53^{\prime} \quad .13695$

$54^{\prime} \quad .13732$

$55^{\prime} \quad .137(69)$

$56^{\prime} \quad .13806$

$57^{\prime} \quad .138+3$

$58^{\prime} .13880$

$59^{\prime} \quad .13917$

$830 \quad 0^{\prime} 983.13953$

${ }^{\prime}$ 13990

$2^{\prime} \quad 1+1127$

$3^{\prime} .1+063$

$4^{\prime} .14099$

$5^{\prime} \quad 1+136$

$6^{\prime} \quad 1+172$

$7^{\prime} .14208$

$8^{\prime} \quad .14244$

$9^{\prime} .14280$

$100^{\prime} \quad 1+316$

II' . 1435I

$12^{\prime} .14387$

$13^{\prime} \quad .1+423$

$14^{\prime} .14458$

$15^{\prime} \quad 14494$

$16^{\prime} \quad 14529$

$17^{\prime} .14564$

$18^{\prime} \quad .14599$

$199^{\prime} \quad .14634$

$200^{\prime} .146(59)$

$21^{\prime} .14704$

$22^{\prime} \quad 14739$

$23^{\prime} .14774$

$24^{\prime} .14808$

$25^{\prime} \quad .148+3$

$26^{\prime} \quad .14877$

$27^{\prime} \quad 14912$

$28^{\prime} \quad .149+6$

$29^{\prime} \quad .14980$

$300^{\prime} .15014$

$31^{\prime} \quad .5048$

$32^{\prime} \quad 15082$

$33^{\prime} \quad 15116$

$34^{\prime}$. 15150

$355^{\prime} .15184$

$36^{\prime} \quad .15217$

$\begin{array}{ll}37^{\prime} & .15251\end{array}$

$38^{\prime} \quad .15284$

$39^{\prime} \quad .15318$

$40^{\prime} \quad .15351$

$t^{\prime} \quad .15384$

$422^{\prime} .15+17$

$43^{\prime} .15450$

$44^{\prime} \quad .15483$

$455^{\prime} \quad .5516$

$466^{\prime} \quad .15549$

$47^{\prime} \quad .15581$

$48^{\prime} \quad .15614$

$49^{\prime} \quad .15646$

$500^{\prime} \quad .15679$

$51^{\prime} .15711$

$52^{\prime} \quad .15743$

$53^{\prime} \quad .15776$

$54^{\prime} .15808$

$55^{\prime} \quad .15840$

$56 i^{\prime} \quad .5871$

$57^{\prime} \quad 15903$

$58^{\prime} \quad .15935$

$59^{\prime} \quad . \quad .5967$ 
Table I - (continuerl)

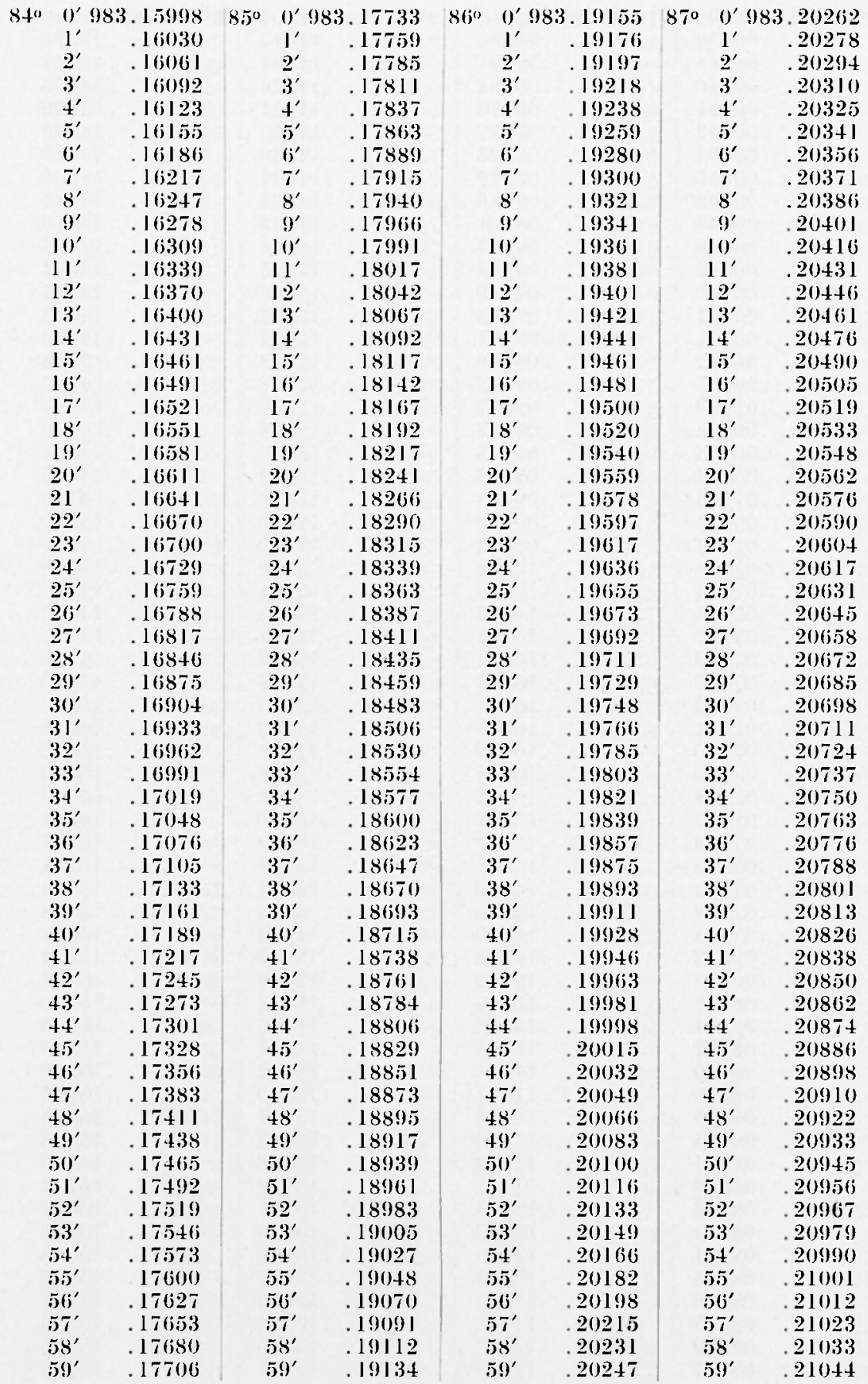


Table $1-($ continued $)$

\begin{tabular}{|c|c|c|c|c|c|c|c|c|c|}
\hline $88^{\circ}$ & .21055 & $88^{\circ} 30$ & 3.21332 & $899^{\circ}$ & $\left(0^{\prime} !\right.$ & .21531 & $899^{\circ}$ & $31^{\prime}$ & 3.21652 \\
\hline$l^{\prime}$ & .21065 & $31^{\prime}$ & .21340 & & $\mathrm{l}^{\prime}$ & .21536 & & $32^{\prime}$ & .21655 \\
\hline $2^{\prime}$ & .21076 & $32^{\prime}$ & .21348 & & $2^{\prime}$ & .21541 & & $33^{\prime}$ & $.216 ; 5$ \\
\hline $3^{\prime}$ & .21086 & $33^{\prime}$ & .21356 & & $3^{\prime}$ & $.215+6$ & & $34^{\prime}$ & .21659 \\
\hline $4^{\prime}$ & .21096 & $34^{\prime}$ & .21363 & & $4^{\prime}$ & .21551 & & 35 & .21662 \\
\hline $\bar{i}^{\prime}$ & .21107 & $35^{\prime}$ & 21371 & & $5^{\prime}$ & $\cdot 21556$ & & $36^{\prime}$ & .21664 \\
\hline $6^{\prime}$ & 21117 & $36^{\prime}$ & .21378 & & $6^{\prime}$ & 21561 & & 37 & .21666 \\
\hline $7^{\prime}$ & .21127 & $37^{\prime}$ & .21386 & & $7^{\prime}$ & $215(35)$ & & $38^{\prime}$ & .21668 \\
\hline $8^{\prime}$ & .21137 & $38^{\prime}$ & .21393 & & $8^{\prime}$ & .21570 & & $39^{\prime}$ & .21670 \\
\hline $9^{\prime}$ & $.211+6$ & $39^{\prime}$ & .21400 & & $\theta^{\prime}$ & 21575 & & $40^{\prime}$ & .21672 \\
\hline $10^{\prime}$ & .21156 & 4()$^{\prime}$ & .21407 & & $10^{\prime}$ & 21579 & & $4 I^{\prime}$ & $.211 ; 73$ \\
\hline $11^{\prime}$ & .21166 & $41^{\prime}$ & .21414 & & $11^{\prime}$ & .21583 & & $42^{\prime}$ & .21675 \\
\hline $12^{\prime}$ & .21175 & $42^{\prime}$ & $.21+21$ & & $12^{\prime}$ & .21588 & & $43^{\prime}$ & .21676 \\
\hline $13^{\prime}$ & $.2118 \pi$ & $43^{\prime}$ & $.21+28$ & & $13^{\prime}$ & .21592 & & $44^{\prime}$ & .21678 \\
\hline $14^{\prime}$ & .21194 & $44^{\prime}$ & $.21+35$ & & $14^{\prime}$ & .21596 & & $45^{\prime}$ & .21679 \\
\hline $15^{\prime}$ & .21203 & $45^{\prime}$ & $.21+41$ & & $15^{\prime}$ & .21600 & & $46^{\prime}$ & .21681 \\
\hline $16^{\prime}$ & .21213 & $46^{\prime}$ & .21448 & & $16^{\prime}$ & .21604 & & $47^{\prime}$ & .21682 \\
\hline $17^{\prime}$ & .21222 & $4 \bar{\prime}^{\prime}$ & .21454 & & $17^{\prime}$ & & & $48^{\prime}$ & .21683 \\
\hline $18^{\prime}$ & .21231 & $48^{\prime}$ & $.21+61$ & & $18^{\prime}$ & .21611 & & $49^{\prime}$ & .21684 \\
\hline $19^{\prime}$ & .21240 & $49^{\prime}$ & .21467 & & $19^{\prime}$ & .21615 & & $50^{\prime}$ & .21685 \\
\hline $20^{\prime}$ & .21249 & $50)^{\prime}$ & $.21+73$ & & $20)^{\prime}$ & .21619 & & $5 l^{\prime}$ & .21686 \\
\hline $21^{\prime}$ & .21257 & 'ו & $.21+79$ & & $2 l^{\prime}$ & .21622 & & $52^{\prime}$ & .21686 \\
\hline $22^{\prime}$ & .21266 & $52^{\prime}$ & $.21+85$ & & $22^{\prime}$ & .21626 & & $533^{\prime}$ & .21687 \\
\hline $23^{\prime}$ & .21275 & $53^{\prime}$ & $.21+91$ & & $23^{\prime}$ & .21629 & & $4^{\prime}$ & .21688 \\
\hline $24^{\prime}$ & .21283 & $5 t^{\prime}$ & 21497 & & $24^{\prime}$ & .21632 & & $55^{\prime}$ & .21688 \\
\hline $25^{\prime}$ & .21292 & $55^{\prime}$ & .215013 & & $25^{\prime}$ & .21635 & & $56^{\prime}$ & .21689 \\
\hline $26^{\prime}$ & .21300 & $566^{\prime}$ & .215099 & & $266^{\prime}$ & .21638 & & $57^{\prime}$ & .21 (6s) \\
\hline $27^{\prime}$ & .21308 & 5 & .21514 & & $27^{\prime}$ & $.216+1$ & & $58^{\prime}$ & 21689 \\
\hline $28^{\prime}$ & .21316 & $58^{\prime}$ & .21520 & & $28^{\prime}$ & .21644 & & $59^{\prime}$ & 2I liss? \\
\hline $29^{\prime}$ & .21324 & $59^{\prime}$ & .21525 & & $29^{\prime}$ & $.21(i 47$ & & & \\
\hline & & & & & $30^{\prime}$ & .21650 & 90 & & 21689 \\
\hline
\end{tabular}

\section{REFERENCES}

(1) Caputo M., The gratity field of the Earlh. Academic Press, Sew YorkJondon, 1) 33 (1967).

${ }^{2}$ ) Captero M., The gracily field of the Earth. Accarlemic Press, New YorkJondon, 1). 32 (196i).

(3) Somliblaxa C., Teoria generale del eampo gravilazonale dell'ellissoide di rolezione. "Mem. Soc. Astr. It." 4, (1927).

(1) Vennisi F., The total mass of the Earth's Atmosphere "Journ. of Geo. physical Research ", 7l, 385 (196(i)). 